\title{
Minimal set of periods for continuous self-maps of the eight space
}

\author{
Jaume Llibre ${ }^{1 *}$ (D) and Ana Sá ${ }^{2}$
}

\section{"Correspondence:}

jllibre@mat.uab.es

${ }^{1}$ Departament de Matemàtiques, Universitat Autònoma de Barcelona, 08193 Bellaterra, Barcelona, Spain Full list of author information is available at the end of the article

\section{Springer}

\begin{abstract}
Let $G_{k}$ be a bouquet of circles, i.e., the quotient space of the interval $[0, k]$ obtained by identifying all points of integer coordinates to a single point, called the branching point of $G_{k}$. Thus, $G_{1}$ is the circle, $G_{2}$ is the eight space, and $G_{3}$ is the trefoil. Let $f: G_{k} \rightarrow G_{k}$ be a continuous map such that, for $k>1$, the branching point is fixed.

If $\operatorname{Per}(f)$ denotes the set of periods of $f$, the minimal set of periods of $f$, denoted by $\operatorname{MPer}(f)$, is defined as $\bigcap_{g \simeq f} \operatorname{Per}(g)$ where $g: G_{k} \rightarrow G_{k}$ is homological to $f$.

The sets $\operatorname{MPer}(f)$ are well known for circle maps. Here, we classify all the sets $\operatorname{MPer}(f)$ for self-maps of the eight space.
\end{abstract}

MSC: $37 \mathrm{E} 15$

Keywords: Continuous maps; Periodic orbit; Period; Minimal period; The space in shape of eight

\section{Introduction and statement of the results}

In dynamical systems it is often the case that topological information can be used to study qualitative or quantitative properties of the system. This work deals with the problem of determining the set of periods of the periodic orbits of a map given the homology class of the map.

A finite graph (simply a graph) $G$ is a topological space formed by a finite set of points $V$ (points of $V$ are called vertices) and a finite set of open arcs (called edges) in such a way that each open arc is attached by its endpoints to vertices. An open arc is a subset of $G$ homeomorphic to the open interval $(0,1)$. Note that a finite graph is compact since it is the union of a finite number of compact subsets (the closed edges and the vertices). Notice that a closed edge is homeomorphic either to the closed interval $[0,1]$ or to the circle. It may be either connected or disconnected, and it may have isolated vertices.

The valence of a vertex is the number of edges with the vertex as an endpoint (where the closed edges homeomorphic to a circle are counted twice). The vertices with valence 1 of a connected graph are endpoints of the graph and the vertices with valence larger than 2 are branching points.

Suppose that $f: G \rightarrow G$ is a continuous map, in what follows a graph map. A fixed point of $f$ is a point $x$ in $G$ such that $f(x)=x$. We will call $x$ a periodic point of period $n$ if $x$ is a

(c) The Author(s) 2021. This article is licensed under a Creative Commons Attribution 4.0 International License, which permits use, sharing, adaptation, distribution and reproduction in any medium or format, as long as you give appropriate credit to the original author(s) and the source, provide a link to the Creative Commons licence, and indicate if changes were made. The images or other third party material in this article are included in the article's Creative Commons licence, unless indicated otherwise in a credit line to the material. If material is not included in the article's Creative Commons licence and your intended use is not permitted by statutory regulation or exceeds the permitted use, you will need to obtain permission directly from the copyright holder. To view a copy of this licence, visit http://creativecommons.org/licenses/by/4.0/. 
fixed point of $f^{n}$ but it is not fixed by any $f^{k}$ for $1 \leq k<n$. We denote by $\operatorname{Per}(f)$ the set of natural numbers corresponding to periods of the periodic points of $f$.

Let $G$ be a connected graph, and let $f$ be a graph map. Then $f$ induces endomorphisms $f_{* k}: H_{k}(G) \rightarrow H_{k}(G)$ for $k=0,1$ on the integral homology groups of $G$, where $H_{0}(G) \approx \mathbb{Z}$ (because $G$ is connected), and $H_{1}(G) \approx \mathbb{Z} \oplus \cdots \oplus \mathbb{Z}$, where $k$ is the number of independent circuits or loops of $G$ as elements of $H_{1}(G)$. A circuit of $G$ is a subset of $G$ homeomorphic to the circle. The endomorphisms $f_{* 0}$ and $f_{* 1}$ are represented by integer matrices. Furthermore, since $G$ is connected, $f_{* 0}$ is the identity.

The endomorphism $f_{* 1}$ will play the main role in our analysis of the minimal sets of periods for graph maps on $G$. In what follows $f_{* 1}$ will be denoted by $f_{*}$. For example, if $H_{1}(G) \approx \mathbb{Z} \oplus \mathbb{Z}$ and

$$
f_{*}=\left(\begin{array}{ll}
a & b \\
c & d
\end{array}\right),
$$

this means that the graph $G$ has two independent oriented circuits. Moreover, if the first circuit covers itself exactly $a_{1}$ times following the same orientation (not necessarily in a consecutive way) and exactly $a_{2}$ times following the converse orientation (not necessarily in a consecutive way), then $a=a_{1}-a_{2}$. Similarly, if the first circuit covers the second one exactly $b_{1}$ times following the same orientation (not necessarily in a consecutive way) and exactly $b_{2}$ times following the converse orientation (not necessarily in a consecutive way), then $b=b_{1}-b_{2}$. An analogous explanation can be given with the second independent circuit and with $b$ and $d$ instead of $a$ and $c$, respectively.

Let $G_{k}$ be a bouquet of $k$ circles, that is, the quotient space of $[0, k]$ obtained by identifying all points of integer coordinates to a single point. Notice that $G_{1}$ is the circle and that $G_{2}$ is usually called the eight space. For the $G_{k}$ graph, we have $H_{0}\left(G_{k}\right) \approx \mathbb{Z}$, $H_{1}\left(G_{k}\right) \approx \mathbb{Z} \oplus \cdots \oplus \mathbb{Z}, f_{* 0} \approx \mathrm{id}$, and $f_{* 1}=f_{*}=A$, where $A$ is a $k \times k$ integral matrix. For more details on graph maps, see [16] or [18].

Our main goal is to study the set $\operatorname{Per}(f)$ for graph maps. More explicitly, we want to provide a description of the minimal set of periods (see below) attained within the homology class of a given graph map. When the map $g: G \rightarrow G$ is homological to $f$ (i.e., $g$ induces the same endomorphisms as $f$ on the homology groups of $G$ ), we shall write $g \simeq f$. We define the minimal set of periods of $f$ to be the set

$$
\operatorname{MPer}(f)=\bigcap_{g \simeq f} \operatorname{Per}(g) .
$$

From its definition $\operatorname{MPer}(f)$ is the maximal subset of periods contained in $\operatorname{Per}(g)$ for all $g \simeq f$.

Our main objective is to characterize the minimal sets of periods MPer $(f)$ for graph maps $f: G_{i} \rightarrow G_{i}$ with the branching point a fixed point for $i=2,3$. So, always $1 \in \operatorname{MPer}(f)$. Even for circle maps $f: G_{1} \rightarrow G_{1}$ the characterization of all minimal sets of periods $\operatorname{MPer}(f)$ is interesting and nontrivial, see Theorem A. This result was stated by Efremova [12] and Block et al. [9] without giving a complete proof. As far as we know, the first complete proof was given in [4].

We denote by $\mathbb{N}$ the set of all natural numbers and by $k \mathbb{N}$ the set $\{k l: l \in \mathbb{N}\}$. 
Theorem A Letf $: G_{1} \rightarrow G_{1}$ be a circle map such that the endomorphism induced by $f$ on the first homology group is $f_{*}=(d)$ (i.e., $d$ is the degree off). Then the following statements hold:

(a) If $d \notin\{-2,-1,0,1\}$, then $\operatorname{MPer}(f)=\mathbb{N}$.

(b) If $d=-2$, then $\operatorname{MPer}(f)=\mathbb{N} \backslash\{2\}$.

(c) If $d \in\{-1,0\}$, then $\operatorname{MPer}(f)=\{1\}$.

(d) If $d=1$, then $\operatorname{MPer}(f)=\emptyset$.

In the next theorem we characterize the minimal sets of periods for eight maps, i.e., for continuous maps $f: G_{2} \rightarrow G_{2}$.

Theorem B Letf $: G_{2} \rightarrow G_{2}$ be an eight map such that

$$
f_{*}=\left(\begin{array}{ll}
a & b \\
c & d
\end{array}\right) .
$$

Suppose that the branching point is a fixed point. Then the following statements hold:

(a) If $\{a, d\} \not \subset\{-2,-1,0,1\}$, then $\operatorname{MPer}(f)=\mathbb{N}$.

(b) If $-2 \in\{a, d\}$ and $\{a, d\} \subset\{-2,-1,0,1\}$, then

$$
\operatorname{MPer}(f)= \begin{cases}\mathbb{N} \backslash\{2\} & \text { if } b c=0, \\ \mathbb{N} & \text { if } b c \neq 0 .\end{cases}
$$

(c) Assume that $\{a, d\} \subset\{-1,0,1\}$.

(c1) If $|a|+|d|=2$, then

$$
\operatorname{MPer}(f)= \begin{cases}\{1\} & \text { if } b c=0, \\ \mathbb{N} \backslash\{2\} & \text { if } b c=1, \\ \mathbb{N} & \text { if } b c=-1 \text { or }|b c|>1 .\end{cases}
$$

(c2) If $|a|+|d|=1$ and

(c21) $a=1, d=0$, then

$$
\operatorname{MPer}(f)= \begin{cases}\{1\} & \text { if } b c=0, \\ \mathbb{N} \backslash\{2\} & \text { if }(b, c) \in R \\ \mathbb{N} & \text { otherwise }\end{cases}
$$

where $R=\{(1,1),(-1,-1),(1,2),(-1,-2)\}$.

(c22) $a=0, d=1$, then it follows (c21) interchanging $b$ and $c$.

(c23) $a=-1, d=0$, then

$$
\operatorname{MPer}(f)= \begin{cases}\{1\} & \text { if } b c=0, \\ \mathbb{N} \backslash\{2\} & \text { if }(b, c) \in R, \\ \mathbb{N} \backslash\{3\} & \text { if } b c=-1, \\ \mathbb{N} & \text { otherwise. }\end{cases}
$$

(c24) $a=0, d=-1$, then it follows (c23) interchanging $b$ and $c$. 
(c3) If $|a|+|d|=0$, then

$$
\operatorname{MPer}(f)= \begin{cases}\{1\} & \text { if } b c=0 \text { or } b c=1, \\ \{1,2\} & \text { if } b c=-1, \\ \{1\} \cup(2 \mathbb{N} \backslash\{2\}) & \text { if } b c=2, \\ \{1\} \cup(2 \mathbb{N} \backslash\{4\}) & \text { if } b c=-2, \\ \{1\} \cup 2 \mathbb{N} & \text { if }|b c|>2 .\end{cases}
$$

We remark that Theorem B implies Theorem A if $f$ has a fixed point by choosing, for instance, $a=b=c=0$.

The study of the minimal set of periods of a homotopy class of maps instead of its homology class is the main objective of the fixed point theory, see for instance the books of Brown [10], Jiang [13], and Kiang [15]. Other extensions from circle maps to $n$-dimensional torus have been done in [2] and [14], and from circle maps to transversal $n$-sphere maps in [11]. Some different results on the periods of graph maps have been given in $[1,3,5-8,16,17]$.

Finally, we recall that the classification of the dynamics on the graph maps helps the classification of the homeomorphisms on the surfaces, see for instance [19] and the references quoted therein.

This work is organized as follows. How to obtain a given period for a graph map by using the notion of $f$-covering is described in Sect. 2. The proof of Theorem B is given in Sect. 3.

\section{Periods and $f$-covering}

Let $f: G \rightarrow G$ be a graph map and $x \in G$ be a periodic point of period $n$. The set $\left\{x, f(x), \ldots, f^{n-1}(x)\right\}$ is called the periodic orbit of $x$.

A set $I \subset G$ will be called an interval if there is a homeomorphism $h: J \rightarrow I$, where $J$ is $[0,1],(0,1],[0,1)$, or $(0,1)$. The set $h((0,1))$ will be called the interior of $I$. If $J=[0,1]$, the interval $I$ will be called closed; if $J=(0,1)$, it will be called open. Notice that it may happen that the above terminology does not coincide with the one used when we think about $I$ as a subset of $G$ (the same applies to the edges of $G$ ). For example, if $G=I=[0,1]$ and $h=$ identity, then for $I$ regarded as a subset of the topological space $G, I$ is both open and closed, and the interior of $I$ is $I$.

Let $C_{i}$ and $C_{j}$ be two circuits of $G_{k}$. A closed interval $I=[u, v]$ is basic if $I \subset C_{i}, f(I)=C_{j}$, where $\{i, j\} \subset\{1,2, \ldots, k\}, f(u)=f(v)=p$, where $p$ is the branching point of $G_{k}$, and there is no other closed interval $K \varsubsetneqq I$ such that $f(K)=C_{j}$. Of course the definition of basic interval also applies to the particular case that $[u, v]=C_{i}$. Let $I$ and $J$ be two basic intervals, $K \subset I$, $L \subset J$ be two subintervals. Then we say that $K f$-covers $L$, and we write $K \rightarrow L$ if there exists a closed subinterval $M$ of $K$ such that $f(M)=L$. If $L=J=C_{j}$, we say that $K=I f$-covers $L$ because either $f(K)=L$ or $K=I=C_{i}$ and $f(K)=L$ by the definition of basic intervals.

Lemma 2.1 Suppose that $I_{1}, I_{2}, \ldots, I_{n}$ are intervals such that $I_{1} \rightarrow I_{2} \rightarrow \cdots \rightarrow I_{n} \rightarrow I_{1}$ with $I_{1}$ different from a circuit. Then there is a fixed point $z$ of $f^{n}$ such that $z \in I_{1}, f(z) \in$ $I_{2}, \ldots, f^{n-1}(z) \in I_{n}$.

Proof Since $I_{n} \rightarrow I_{1}$ and $I_{1}$ is not a circuit, there is a closed interval $J_{n} \subset I_{n}$ such that $f\left(J_{n}\right)=$ $I_{1}$. Similarly, there are closed intervals or circuits $J_{1}, \ldots, J_{n-1}$ such that, for each $k=1, \ldots, n-$ 
$1, J_{k} \subset I_{k}$ and $f\left(J_{k}\right)=J_{k+1}$. It follows that $f^{n}\left(J_{1}\right)=I_{1}$ and since $J_{1} \subset I_{1}$ and $I_{1}$ is not a circuit, by Bolzano's theorem $f^{n}$ has a fixed point $z \in J_{1}$. Clearly, $z \in I_{1}, f(z) \in I_{2}, \ldots, f^{n-1}(z) \in I_{n}$.

A sequence of the form $I_{1} \rightarrow I_{2} \rightarrow \cdots \rightarrow I_{n} \rightarrow I_{1}$ is called a loop of length $n$. Let $I_{1} \rightarrow I_{2} \rightarrow \cdots \rightarrow I_{n} \rightarrow I_{1}$ and $J_{1} \rightarrow J_{2} \rightarrow \cdots \rightarrow J_{m} \rightarrow J_{1}$ be two loops such that $I_{1}=J_{1}$. We define the concatenation of these two loops as the loop $I_{1} \rightarrow I_{2} \rightarrow \cdots \rightarrow I_{n} \rightarrow I_{1} \rightarrow$ $J_{2} \rightarrow \cdots \rightarrow J_{m} \rightarrow I_{1}$. We say that a loop is an $m$-repetition, $m \geq 2$, of a given loop if it is the concatenation of that loop with itself $m$ times. We say that a loop is non-repetitive if it is not an $m$-repetition of any of its subloops with $m \geq 2$.

In what follows, a $G_{k}$-map $f$ is a continuous map $f: G_{k} \rightarrow G_{k}$ such that $f(p)=p$, where $p$ is the branching point of $G_{k}$.

Proposition 2.2 Let $f$ be a $G_{k}$-map. Suppose that $f$ has two intervals $I_{1}$ and $I_{2}$ such that $\operatorname{Int}\left(I_{1}\right) \cap \operatorname{Int}\left(I_{2}\right)=\emptyset$ and $I_{1} \cap I_{2}$ has no fixed points. Iff has the subgraph $\odot I_{1} \rightleftarrows I_{2} \ominus$, then $\operatorname{Per}(f)=\mathbb{N}$.

Proof Clearly, since $p \notin I_{1} \cap I_{2}$, at least one of the intervals, $I_{1}$ or $I_{2}$, is not a circuit. Without loss of generality, we assume that $I_{1}$ is not a circuit. We consider the non-repetitive loop $I_{1} \rightarrow I_{2} \rightarrow I_{1} \rightarrow \cdots \rightarrow I_{1}$ of length $n \geq 2$. Since $\operatorname{Int}\left(I_{1}\right) \cap \operatorname{Int}\left(I_{2}\right)=\emptyset$ and $I_{1} \cap I_{2}$ has no fixed points, by Lemma 2.1, there is a periodic point $z$ of $f$ with period $n \geq 2$. That is, $\operatorname{Per}(f)=\mathbb{N}$.

In what follows when we say "we have two intervals $A$ and $B$ " we are really saying that we have two different intervals $A$ and $B$. We remark that if we have two basic intervals $I_{1}$ and $I_{2}$ such that $p \notin I_{1} \cap I_{2}$, then they satisfy the assumptions of Proposition 2.2.

Proposition 2.3 Letf be a $G_{k}$-map. Suppose that $f$ has three intervals $I_{1}, I_{2}$, and $I_{3}$ such that $\operatorname{Int}\left(I_{i}\right) \cap \operatorname{Int}\left(I_{j}\right)=\emptyset$ for all $i \neq j$ and $I_{i} \cap I_{j}$ has no fixed points for some $i \neq j$. If $f$ has the subgraph $\odot I_{1} \rightarrow I_{2} \rightarrow I_{3} \rightarrow I_{1}$, then $\operatorname{Per}(f) \supset \mathbb{N} \backslash\{2\}$. Moreover, if $I_{2} \cap I_{3}=\emptyset$ and $I_{3} \rightarrow I_{2}$, then $2 \in \operatorname{Per}(f)$.

Proof We consider the non-repetitive loop $I_{1} \rightarrow I_{2} \rightarrow I_{3} \rightarrow I_{1} \rightarrow \cdots \rightarrow I_{1}$ of length $n \geq$ 3. Since $\operatorname{Int}\left(I_{i}\right) \cap \operatorname{Int}\left(I_{j}\right)=\emptyset$ for all $i \neq j$ and $I_{i} \cap I_{j}$ has no fixed points for some $i \neq j$, by Lemma 2.1, there is a periodic point $z$ of $f$ with period $n \geq 3$. Therefore, $\operatorname{Per}(f) \supset \mathbb{N} \backslash\{2\}$.

We suppose now that $I_{2} \cap I_{3}=\emptyset$ and $I_{3} \rightarrow I_{2}$. We consider the non-repetitive loop $I_{2} \rightarrow$ $I_{3} \rightarrow I_{2}$ of length 2 . By Lemma 2.1 there is a periodic point $z$ of $f$ with period 2 .

We remark that if we have three basic intervals $I_{1}, I_{2}$, and $I_{3}$ such that $p \notin I_{i}$ for some $i \in\{1,2,3\}$, then we are in the assumptions of Proposition 2.3.

\section{The eight}

In this section we shall prove Theorem B. The two circuits of $G_{2}$ are denoted by $C_{1}$ and $C_{2}$. If $f_{*}$ is given as in Theorem B, we consider that the circuit $C_{1}$ covers itself $|a|$ times and it covers $C_{2}|c|$ times. Similarly for the circuit $C_{2}$.

Proof of statement (a) of Theorem B Suppose that $\{a, d\} \not \subset\{-2,-1,0,1\}$.

Case 1: Assume that $\{\mathbf{a}, \mathbf{d}\} \not \subset\{-\mathbf{2},-\mathbf{1}, \mathbf{0}, \mathbf{1}, \mathbf{2}\}$. Without loss of generality, we may assume that $|a| \geq 3$. From the graph of $f$ (see for instance Fig. 1), it is clear that there are two basic 
(a) $a=3, d=-1$

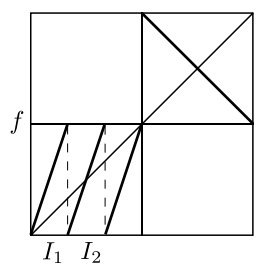

(b) $a=3, d=-1$

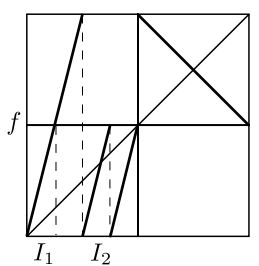

(c) $a=-3, d=0$

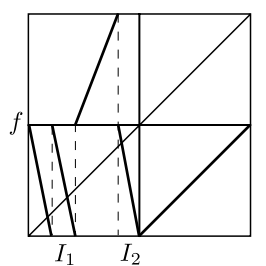

Figure 1 Examples of maps with $\{a, d\} \not \subset\{-2,-1,0,1,2\}$

Figure $2 l_{1_{1}}=\left[p, a_{1}\right], l_{21}=\left[b_{0}, b_{1}\right]$, and $l_{1_{3}}=\left[a_{1}, a_{2}\right]$

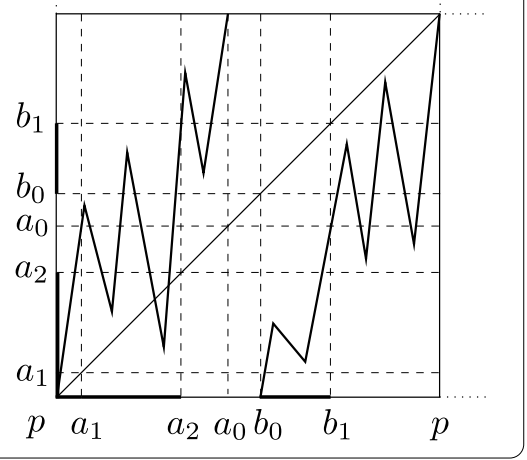

intervals $I_{1}$ and $I_{2}$ in $C_{1}$ such that $p \notin I_{1} \cap I_{2}$ and $f$ has the subgraph of Proposition 2.2, so $\operatorname{Per}(f)=\mathbb{N}$. That is, $\operatorname{MPer}(f)=\mathbb{N}$.

Case 2: Suppose that $\mathbf{2} \in\{\mathbf{a}, \mathbf{d}\}$ and $\{\mathbf{a}, \mathbf{d}\} \subset\{-\mathbf{2},-\mathbf{1}, \mathbf{0}, \mathbf{1}, \mathbf{2}\}$. Without loss of generality, we may assume that $a=2$.

Since $a=2$, this means that $f$ has at least two basic intervals $I_{1}$ and $I_{2}$ in $C_{1}$ such that $f$ has the subgraph of Proposition 2.2. If $p \notin I_{1} \cap I_{2}$, then, by Proposition 2.2, $\operatorname{Per}(f)=\mathbb{N}$. But not always $I_{1}$ and $I_{2}$ satisfy that $p \notin I_{1} \cap I_{2}$. In this case let $p$ and $a_{0}$ be the endpoints of $I_{1}$, $b_{0}$ and $p$ be the endpoints of $I_{2}$ (see for instance Fig. 2).

We establish an ordering in the intervals $I_{1}$ and $I_{2}$ in such a way that $p$ is the smallest element of $I_{1}$ and the greatest of $I_{2}$. Set $I_{1}=\left[p, a_{0}\right]$ and $I_{2}=\left[b_{0}, p\right]$. Notice that we may have $a_{0}=b_{0}$. Consider the subset $\left(f \mid I_{1}\right)^{-1}\left(a_{0}\right)$ of $C_{1}$. Let $a_{1}$ be the infimum of the points in $\left(f \mid I_{1}\right)^{-1}\left(a_{0}\right)$. Consider the subset $\left(f \mid I_{2}\right)^{-1}\left(a_{0}\right)$ of $C_{1}$ and choose $b_{1}$ to be the infimum of the points in $\left(f \mid I_{2}\right)^{-1}\left(a_{0}\right)$. Set $I_{1_{1}}=\left[p, a_{1}\right], I_{1_{2}}=\left[a_{1}, a_{0}\right]$, and $I_{2_{1}}=\left[b_{0}, b_{1}\right]$. Now we take the interval $I_{1_{3}}=\left[a_{1}, a_{2}\right]$, where $a_{2}$ denotes the infimum of the points in the subset $\left(f \mid I_{1_{2}}\right)^{-1}\left(b_{1}\right)$ of $C_{1}$. Then $f$ has the subgraph $\curvearrowright I_{1_{1}} \rightarrow I_{1_{3}} \rightleftarrows I_{2_{1}} \rightarrow I_{1_{1}}$. Since $I_{2_{1}} \cap I_{1_{3}}=\emptyset$, by Proposition 2.3, $n \in \operatorname{Per}(f)$ for all $n \geq 1$. Therefore, $\operatorname{MPer}(f)=\mathbb{N}$. This proves statement (a).

Proof of statement (b) of Theorem B Suppose that $-\mathbf{2} \in\{\mathbf{a}, \mathbf{d}\}$ and $\{\mathbf{a}, \mathbf{d}\} \subset\{-\mathbf{2},-\mathbf{1}, \mathbf{0}, \mathbf{1}\}$. Without loss of generality, we may assume that $\mathbf{a}=\mathbf{- 2}$. 
First we suppose that $\mathbf{b c} \neq \mathbf{0}$. We always have four basic intervals $I_{1}, I_{2}, I_{3}$, and $I_{4}$, $I_{1}, I_{2}, I_{3} \subset C_{1}$, and $I_{4} \subset C_{2}$ such that either $p \notin I_{1} \cap I_{3}$ or $I_{2} \cap I_{4}=\emptyset$ and $f$ has the subgraph

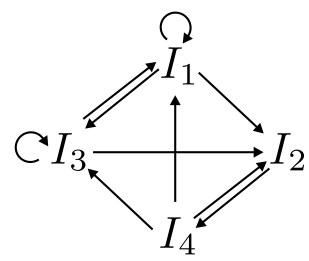

(see for instance Fig. 3).

If $p \notin I_{1} \cap I_{3}$, by Proposition 2.2, $\operatorname{Per}(f)=\mathbb{N}$. If $I_{2} \cap I_{4}=\emptyset$, by $\operatorname{Proposition} 2.3, \operatorname{Per}(f)=\mathbb{N}$. Therefore, if $b c \neq 0, \operatorname{MPer}(f)=\mathbb{N}$.

We suppose now that $\mathbf{b c}=\mathbf{0}$. As it can be deduced from the examples of Fig. $4,2 \notin$ $\operatorname{MPer}(f)$.

Since $a=-2$, this means that $f$ has at least two basic intervals $I_{1}$ and $I_{2}$ in $C_{1}$ such that $f$ has the subgraph of Proposition 2.2. If $p \notin I_{1} \cap I_{2}$, then by Proposition 2.2 $\operatorname{Per}(f)=\mathbb{N}$. But not always $p \notin I_{1} \cap I_{2}$. In this case let $p$ and $a_{0}$ be the endpoints of $I_{1}, b_{0}$ and $p$ be the endpoints of $I_{2}$ (see for instance Fig. 5). We consider an ordering in the intervals $I_{1}$ and $I_{2}$ in such a way that $p$ is the smallest element of $I_{1}$ and the greatest of $I_{2}$. Write $I_{1}=\left[p, a_{0}\right]$ and $I_{2}=\left[b_{0}, p\right]$. Notice that we may have $a_{0}=b_{0}$. Consider the subsets $\left(f \mid I_{1}\right)^{-1}\left(a_{0}\right)$ and $\left(f \mid I_{2}\right)^{-1}\left(a_{0}\right)$ of $C_{1}$. Let $a_{1}$ be the infimum of the points in $\left(f \mid I_{1}\right)^{-1}\left(a_{0}\right)$ and $b_{1}$ be the infimum

(a) $a=-2, d=0, b c=1$

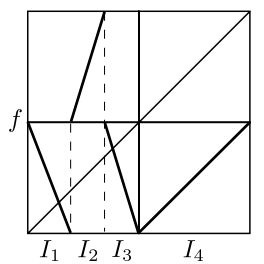

(b) $a=-2, d=1, b c=1$

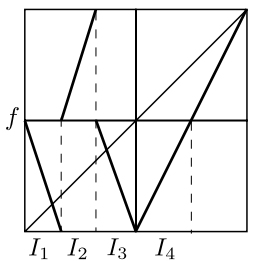

(c) $a=-2, d=-1, b c=-1$

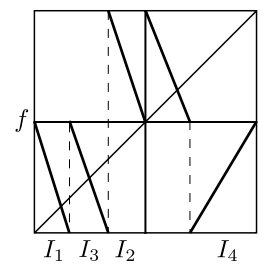

Figure 3 Examples of maps with $a=-2$ and $b c \neq 0$
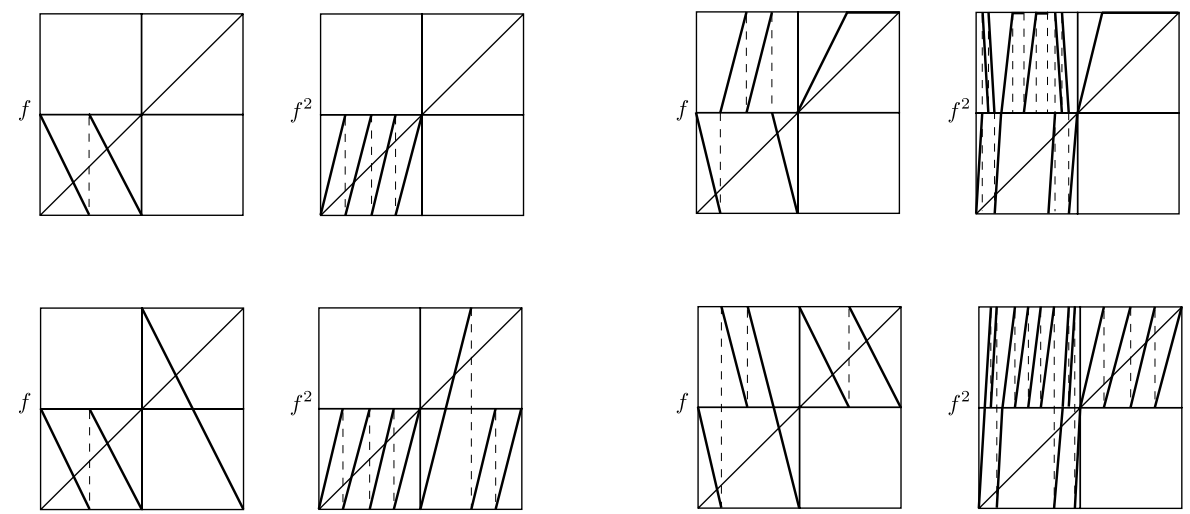

Figure 4 Examples of maps with $a=-2, d \in\{-2,-1,0,1\}, b c=0$, and $2 \notin \operatorname{Per}(f)$ 
Figure $5 l_{1_{1}}=\left[p, a_{1}\right], I_{1_{2}}=\left[a_{1}, a_{0}\right]$, and $I_{21}=\left[b_{1}, p\right]$

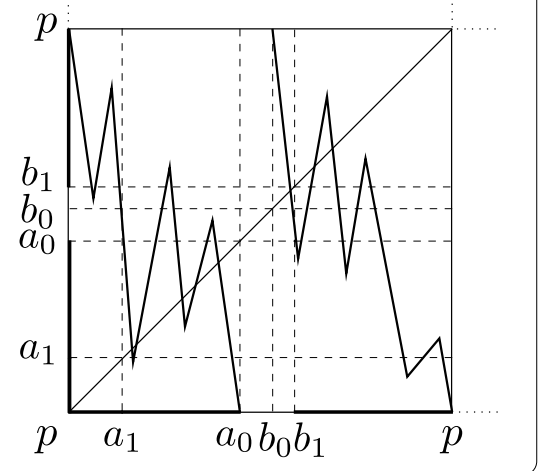

Figure 6 Examples of maps with $\{a, d\} \subset\{-1,0,1\}$, $|a|+|d|=2$, and $b c=0$
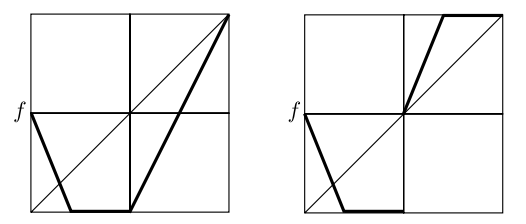

of the points in $\left(f \mid I_{2}\right)^{-1}\left(a_{0}\right)$. Set $I_{1_{1}}=\left[p, a_{1}\right], I_{1_{2}}=\left[a_{1}, a_{0}\right]$, and $I_{2_{1}}=\left[b_{1}, p\right]$. Then $f$ has the subgraph $\odot I_{1_{2}} \rightarrow I_{1_{1}} \rightarrow I_{2_{1}} \rightarrow I_{1_{2}}$. Since we are in the assumptions of Proposition 2.3, $n \in \operatorname{Per}(f)$ for all $n \neq 2$. Therefore, $\operatorname{MPer}(f)=\mathbb{N} \backslash\{2\}$. This proves statement (b).

Proof of statement (c1) of Theorem B Suppose that $\{\mathbf{a}, \mathbf{d}\} \subset\{-\mathbf{1}, \mathbf{0}, \mathbf{1}\}$ and $|\mathbf{a}|+|\mathbf{d}|=\mathbf{2}$. We consider first the case $\mathbf{b c}=\mathbf{0}$. Without loss of generality, we may assume that $c=0$. From the examples of Fig. 6 it is clear that $n \notin \operatorname{MPer}(f)$ for any $n \in \mathbb{N}$ larger than 1 , so $\operatorname{MPer}(f)=$ $\{1\}$ since the branching is fixed.

We assume now that $|\mathbf{b c}|>\mathbf{1}$. From the graph of $f$ (see for instance Fig. 7) it is easy to see that we always have three basic intervals $I_{1}, I_{2}$, and $I_{3}$, with $I_{1}, I_{2} \subset C_{1}$ and $I_{3} \subset C_{2}$ such that $p \notin I_{i}$ for some $i \in\{1,2,3\}$ and $f$ has the subgraph of Proposition 2.3, so $\operatorname{Per}(f) \supset \mathbb{N} \backslash\{2\}$. Now we will prove that $2 \in \operatorname{MPer}(f)$.

If $\{\mathbf{b}, \mathbf{c}\} \not \subset\{-\mathbf{2}, \mathbf{- 1}, \mathbf{1}, \mathbf{2}\}$, that is, if either $|b| \geq 3$ or $|c| \geq 3$, we can choose $I_{2}$ in one circuit and $I_{3}$ in the other circuit in such a way that $I_{2} \cap I_{3}=\emptyset$ (see (a), (b), and (c) of Fig. 7) and $I_{3} \rightarrow I_{2}$. By Proposition 2.3, $2 \in \operatorname{Per}(f)$. If $\{\mathbf{b}, \mathbf{c}\} \subset\{-\mathbf{2},-\mathbf{1}, \mathbf{1}, \mathbf{2}\}$, in general there do not exist two basic intervals $I_{i}$ and $I_{j}, I_{i} \neq I_{j}$, such that $p \notin I_{i} \cap I_{j}$ and $I_{i} \rightleftarrows I_{j}$ (see (e) and (f) of Fig. 7). If they exist, then by Lemma 2.1, considering the non-repetitive loop $I_{i} \rightarrow I_{j} \rightarrow I_{i}$, there is a periodic point $z$ of $f$ with period 2. If they do not exist, we shall find two intervals with empty intersection such that one $f$-covers the other.

We suppose first that $|b c|=2$. We may assume, without loss of generality, that $|b|=1$ and $|c|=2$. We know that $f$ has five basic intervals $I_{1}, I_{2}, I_{3}, I_{4}$, and $I_{5}$, the first three in $C_{1}$ and the other two in $C_{2}$, such that $f\left(I_{2}\right)=f\left(I_{3}\right)=f\left(I_{5}\right)=C_{2}$ and $f\left(I_{1}\right)=f\left(I_{4}\right)=C_{1}$. Let $p$ and $a_{0}$ be the endpoints of $I_{2}, a_{0}$ and $a_{1}$ be the endpoints of $I_{1}, a_{1}$ and $p$ be the endpoints of $I_{3}$ (see for instance Fig. 8).

We consider an ordering in the intervals $I_{1}, I_{2}$, and $I_{3}$ in such a way that $p$ is the smallest element of $I_{2}$ and the greatest of $I_{3}$. Set $I_{2}=\left[p, a_{0}\right], I_{1}=\left[a_{0}, a_{1}\right]$, and $I_{3}=\left[b_{0}, p\right]$. We have two possibilities for the interval $I_{4}$ : either $I_{4}=\left[p, b_{0}\right]$ or $I_{4}=\left[b_{0}, p\right]$. If $I_{4}=\left[p, b_{0}\right]$ and $b=1$, let $b_{1}$ be the supremum of the points in $\left(f \mid I_{4}\right)^{-1}\left(a_{1}\right)$ and $I_{4_{2}}=\left[b_{1}, b_{0}\right]$. We have $I_{4_{2}} \rightleftarrows I_{3}$ and 
(a) $a=1, d=1, b c=-3$

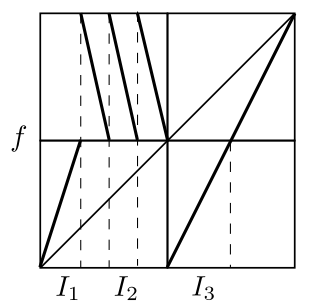

(d) $a=-1, d=-1, b c=-2$

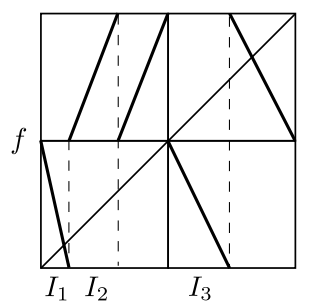

(b) $a=-1, d=1, b c=-3$

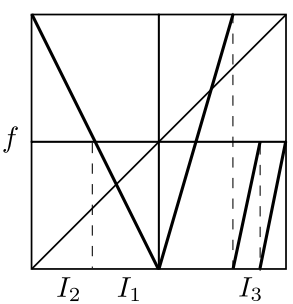

(e) $a=-1, d=-1, b c=-2$

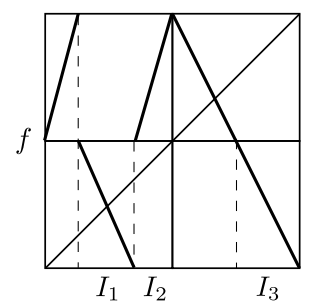

(c) $a=1, d=1, b c=6$

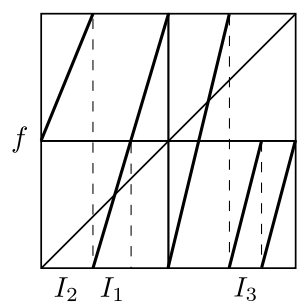

(f) $a=1, d=1, b c=-4$

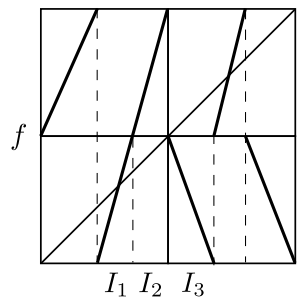

Figure 7 Examples of maps with $\{a, d\} \subset\{-1,0,1\},|a|+|d|=2$, and $|b c|>1$

(a)

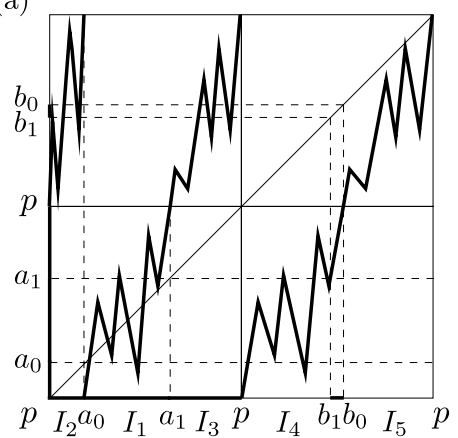

(c)

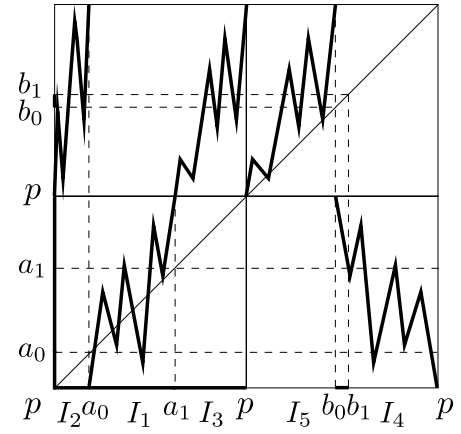

(b)

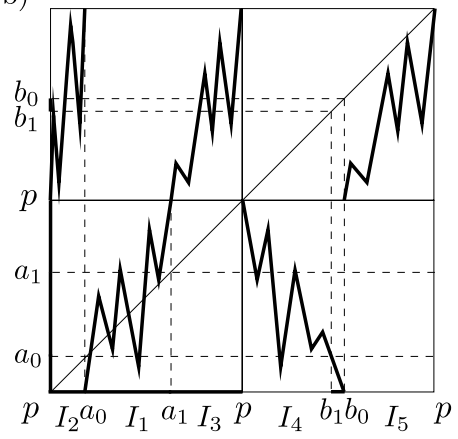

(d)

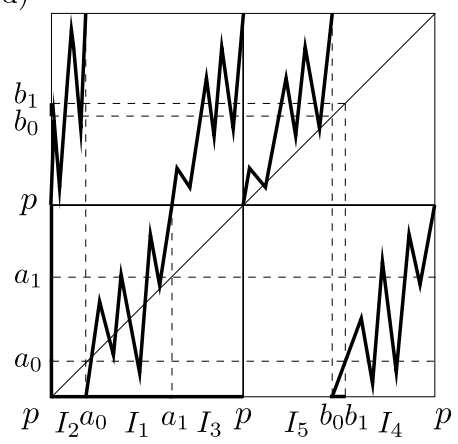

Figure 8 Examples of maps with $\{a, d\} \subset\{-1,0,1\},|a|+|d|=2$ and $|b c|=2$

$I_{4_{2}} \cap I_{3}=\emptyset$, so, by Lemma $2.1,2 \in \operatorname{Per}(f)$. If $I_{4}=\left[p, b_{0}\right]$ and $b=-1$, set $b_{1}=\sup \left\{\left(f \mid I_{4}\right)^{-1}\left(a_{0}\right)\right\}$ and $I_{4_{2}}=\left[b_{1}, b_{0}\right]$. Then $I_{4_{2}} \rightleftarrows I_{2}$ and $I_{4_{2}} \cap I_{2}=\emptyset$ so, by Lemma $2.1,2 \in \operatorname{Per}(f)$. If $I_{4}=\left[b_{0}, p\right]$ and $b=1$, write $b_{1}=\inf \left\{\left(f \mid I_{4}\right)^{-1}\left(a_{0}\right)\right\}$ and $I_{4_{1}}=\left[b_{0}, b_{1}\right]$. Then $I_{4_{1}} \rightleftarrows I_{2}$ and $I_{4_{1}} \cap I_{2}=\emptyset$, so, by 

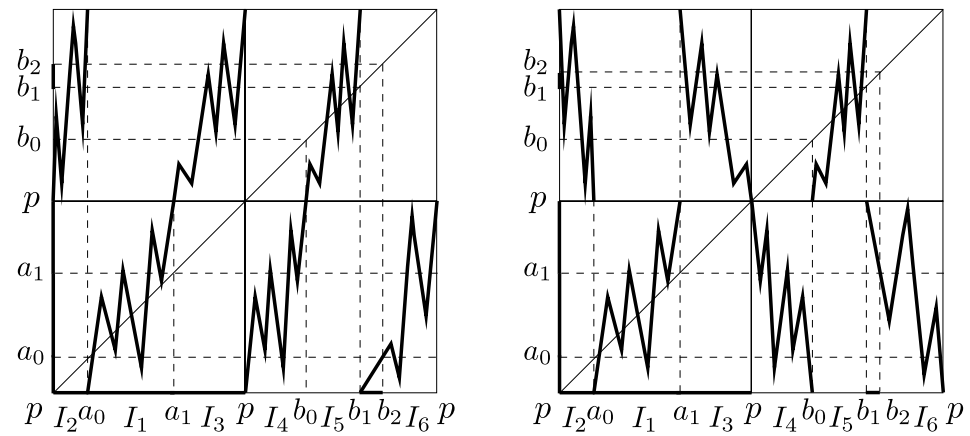

Figure 9 Examples of maps with $\{a, d\} \subset\{-1,0,1\},|a|+|d|=2$, and $|b c|=4$

(a)

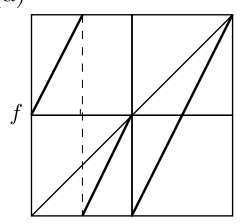

(c)

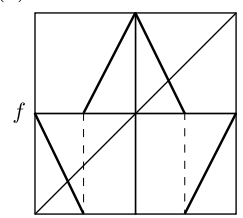

(e)

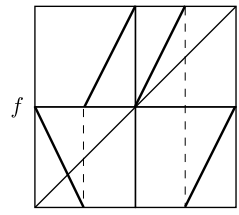

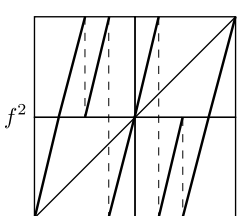
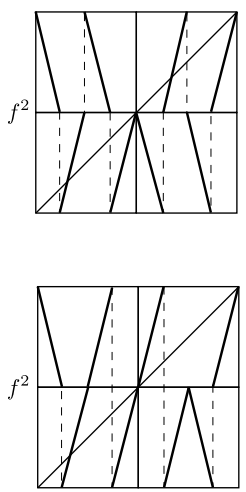

(b)
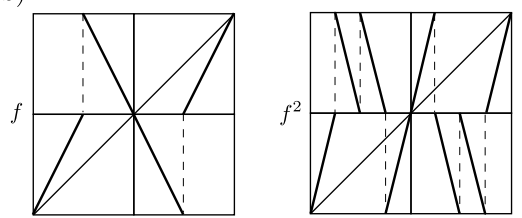

(d)
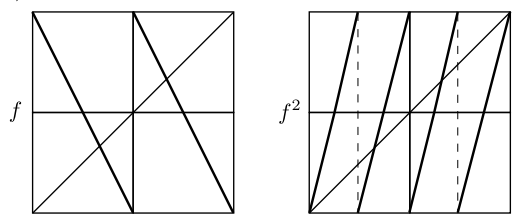

(f)
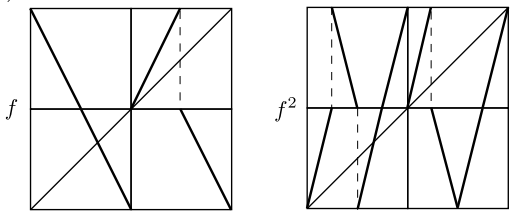

Figure 10 Examples of maps with $\{a, d\} \subset\{-1,0,1\},|a|+|d|=2, b c=1$, and $2 \notin \operatorname{Per}(f)$

Lemma 2.1, $2 \in \operatorname{Per}(f)$. If $I_{4}=\left[b_{0}, p\right]$ and $b=-1$, take $b_{1}=\inf \left\{\left(f \mid I_{4}\right)^{-1}\left(a_{1}\right)\right\}$ and $I_{4_{1}}=\left[b_{0}, b_{1}\right]$. Then $I_{4_{1}} \rightleftarrows I_{3}$ and $I_{4_{1}} \cap I_{3}=\emptyset$, so, by Lemma 2.1, $2 \in \operatorname{Per}(f)$.

Suppose now that $|b c|=4$. We know that $f$ has six basic intervals $I_{1}, I_{2}, I_{3}, I_{4}, I_{5}$, and $I_{6}$, the first three in $C_{1}$ and the other three in $C_{2}$, such that $f\left(I_{2}\right)=f\left(I_{3}\right)=f\left(I_{5}\right)=C_{2}$ and $f\left(I_{1}\right)=f\left(I_{4}\right)=f\left(I_{6}\right)=C_{1}$ (see for instance Fig. 9). Using the same ordering as the above set $I_{2}=\left[p, a_{0}\right], I_{1}=\left[a_{0}, a_{1}\right], I_{3}=\left[b_{0}, p\right], I_{4}=\left[p, b_{0}\right], I_{5}=\left[b_{0}, b_{1}\right]$, and $I_{6}=\left[b_{1}, p\right]$. If $b=2$, set $b_{2}=\inf \left\{\left(f \mid I_{6}\right)^{-1}\left(a_{0}\right)\right\}$ and $I_{6_{1}}=\left[b_{1}, b_{2}\right]$. Then $I_{6_{1}} \rightleftarrows I_{2}$ and $I_{6_{1}} \cap I_{2}=\emptyset$ so, by Lemma $2.1,2 \in$ $\operatorname{Per}(f)$. If $b=-2$, write $b_{2}=\inf \left\{\left(f \mid I_{6}\right)^{-1}\left(a_{1}\right)\right\}$ and $I_{6_{1}}=\left[b_{1}, b_{2}\right]$. Then $I_{6_{1}} \rightleftarrows I_{3}$ and $I_{6_{1}} \cap I_{3}=\emptyset$ so, by Lemma 2.1, $2 \in \operatorname{Per}(f)$. Therefore, if $|b c|>1, \operatorname{MPer}(f)=\mathbb{N}$.

We suppose that $|\mathbf{b c}|=\mathbf{1}$. We assume that $\mathbf{b}=\mathbf{c}=\mathbf{1}$. As it can be seen from examples (a), (c), and (e) of Fig. 10, $2 \notin \operatorname{MPer}(f)$. Now we will prove that $\operatorname{Per}(f)=\mathbb{N} \backslash\{2\}$.

We know that $f$ has four basic intervals $I_{1}, I_{2}, I_{3}$, and $I_{4}$, the first two in $C_{1}$ and the other two in $C_{2}$, such that $f\left(I_{1}\right)=f\left(I_{3}\right)=C_{1}$ and $f\left(I_{2}\right)=f\left(I_{4}\right)=C_{2}$. We have four possibilities for these intervals. Let $a_{0} \in I_{1} \cap I_{2}$ and $b_{0} \in I_{3} \cap I_{4}$ (see for instance Fig. 11). First, we take 


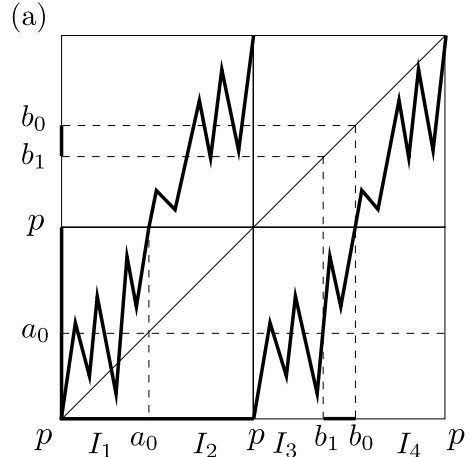

(c)

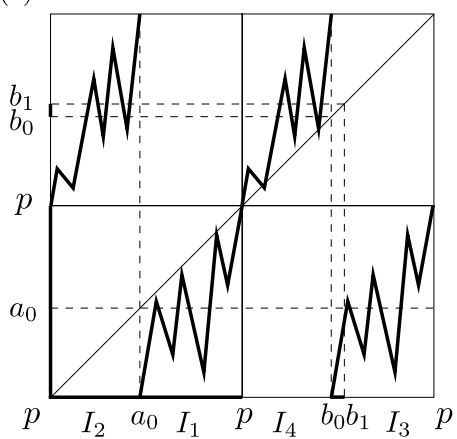

(b)

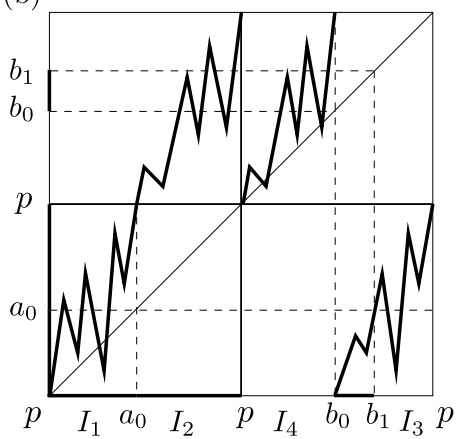

(d)

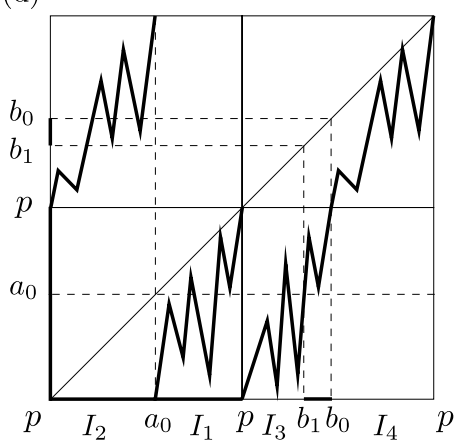

Figure 11 Examples of maps with $\{a, d\} \subset\{-1,0,1\},|a|+|d|=2$, and $b=c=1$

the interval $I_{3}$ to be $\left[p, b_{0}\right]$. Set $I_{3_{2}}=\left[b_{1}, b_{0}\right]$ where $b_{1}=\sup \left\{\left(f \mid I_{3}\right)^{-1}\left(a_{0}\right)\right\}$. If $I_{1}=\left[p, a_{0}\right]$, then $f$ has the subgraph $\odot I_{4} \rightarrow I_{3_{2}} \rightarrow I_{2} \rightarrow I_{4}$, and by Proposition 2.3, $\operatorname{Per}(f)=\mathbb{N} \backslash\{2\}$. If $I_{1}=\left[a_{0}, p\right]$, then $f$ has the subgraph $\triangle_{1} \rightarrow I_{2} \rightarrow I_{3_{2}} \rightarrow I_{1}$, and by Proposition 2.3, $\operatorname{Per}(f)=$ $\mathbb{N} \backslash\{2\}$.

Now we take the interval $I_{3}$ to be $\left[b_{0}, p\right]$. Set $b_{1}=\inf \left\{\left(f \mid I_{3}\right)^{-1}\left(a_{0}\right)\right\}$ and $I_{3_{1}}=\left[b_{0}, b_{1}\right]$. If $I_{1}=\left[p, a_{0}\right]$, then $f$ has the subgraph $\odot I_{1} \rightarrow I_{2} \rightarrow I_{3_{1}} \rightarrow I_{1}$, and by Proposition 2.3, $\operatorname{Per}(f)=$ $\mathbb{N} \backslash\{2\}$. If $I_{1}=\left[a_{0}, p\right]$, then $f$ has the subgraph $\odot I_{4} \rightarrow I_{3_{1}} \rightarrow I_{2} \rightarrow I_{4}$, and by Proposition 2.3, $\operatorname{Per}(f)=\mathbb{N} \backslash\{2\}$. Therefore, if $|a|=|d|=1$ and $b=c=1$, then $\operatorname{MPer}(f)=\mathbb{N} \backslash\{2\}$.

We assume now that $\mathbf{b}=\mathbf{c}=-\mathbf{1}$. As it can be seen from examples (b), (d), and (f) of Fig. 10, $2 \notin \operatorname{MPer}(f)$. Now we will prove that $\operatorname{Per}(f)=\mathbb{N} \backslash\{2\}$.

We know that $f$ has four basic intervals $I_{1}, I_{2}, I_{3}$, and $I_{4}$, the first two in $C_{1}$ and the other two in $C_{2}$, such that $f\left(I_{1}\right)=f\left(I_{3}\right)=C_{1}$ and $f\left(I_{2}\right)=f\left(I_{4}\right)=C_{2}$. We have four possibilities for these intervals. Let $a_{0} \in I_{1} \cap I_{2}$ and $b_{0} \in I_{3} \cap I_{4}$ (see for instance Fig. 12). First we take $I_{3}$ to be the interval $\left[p, b_{0}\right]$. Consider $b_{1}=\sup \left\{\left(f \mid I_{3}\right)^{-1}\left(a_{0}\right)\right\}$ and $I_{3_{2}}=\left[b_{1}, b_{0}\right]$. If $I_{1}=\left[p, a_{0}\right]$, then $f$ has the subgraph $\triangle_{1} \rightarrow I_{2} \rightarrow I_{3_{2}} \rightarrow I_{1}$, and by Proposition 2.3, $\operatorname{Per}(f)=\mathbb{N} \backslash\{2\}$. If $I_{1}=\left[a_{0}, p\right]$, then $f$ has the subgraph $\triangle_{4} \rightarrow I_{3_{2}} \rightarrow I_{2} \rightarrow I_{4}$, and by Proposition 2.3, $\operatorname{Per}(f)=$ $\mathbb{N} \backslash\{2\}$.

If $I_{3}=\left[b_{0}, p\right]$, consider $b_{1}=\inf \left\{\left(f \mid I_{3}\right)^{-1}\left(a_{0}\right)\right\}$ and $I_{3_{1}}=\left[b_{0}, b_{1}\right]$. If $I_{1}=\left[p, a_{0}\right]$, then $f$ has the subgraph $\triangle_{4} \rightarrow I_{3_{1}} \rightarrow I_{2} \rightarrow I_{4}$, and by Proposition 2.3, $\operatorname{Per}(f)=\mathbb{N} \backslash\{2\}$. If $I_{1}=\left[a_{0}, p\right]$, then $f$ has the subgraph $\odot I_{1} \rightarrow I_{2} \rightarrow I_{3_{1}} \rightarrow I_{1}$, and by Proposition 2.3, $\operatorname{Per}(f)=\mathbb{N} \backslash\{2\}$. Therefore, if $b=c=-1$, then $\operatorname{MPer}(f)=\mathbb{N} \backslash\{2\}$. Hence, if $|a|+|d|=2$ and $b c=1, \operatorname{MPer}(f)=$ $\mathbb{N} \backslash\{2\}$. 
(a)

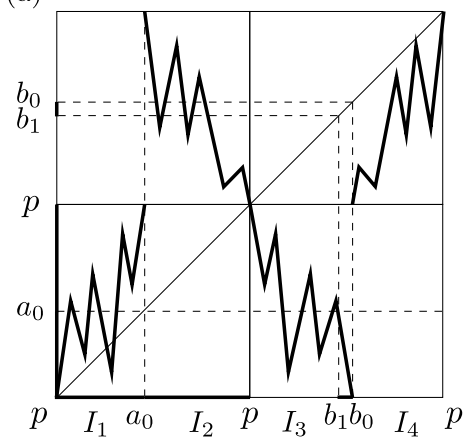

(c)

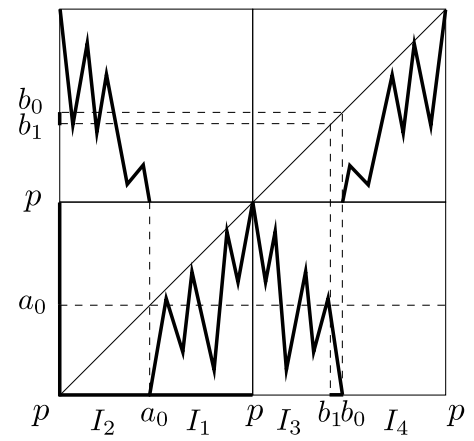

(b)

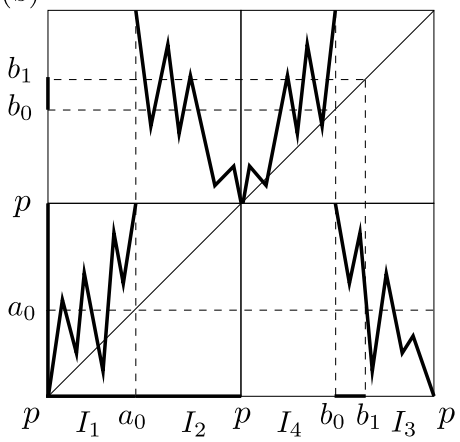

(d)

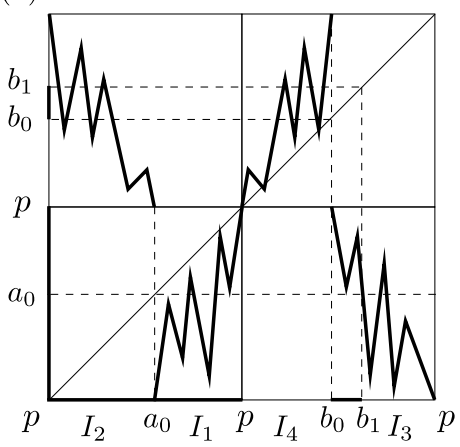

Figure 12 Examples of maps with $\{a, d\} \subset\{-1,0,1\},|a|+|d|=2$, and $b=c=-1$

We consider now the case $\mathbf{b}=-\mathbf{1}$ and $\mathbf{c}=\mathbf{1}$. We know that $f$ has four basic intervals $I_{1}$, $I_{2}, I_{3}$, and $I_{4}$, the first two in $C_{1}$ and the other two in $C_{2}$, such that $f\left(I_{1}\right)=f\left(I_{3}\right)=C_{1}$ and $f\left(I_{2}\right)=f\left(I_{4}\right)=C_{2}$. We have four possibilities for these intervals. Let $a_{0} \in I_{1} \cap I_{2}$ and $b_{0} \in$ $I_{3} \cap I_{4}$ (see for instance Fig. 13). We suppose first that $I_{2}=\left[a_{0}, p\right]$. If $I_{3}=\left[p, b_{0}\right]$, choose $a_{1}=$ $\inf \left\{\left(f \mid I_{2}\right)^{-1}\left(b_{0}\right)\right\}$ and set $I_{2_{1}}=\left[a_{0}, a_{1}\right]$. Then $f$ has the subgraph $\odot I_{1} \rightarrow I_{2_{1}} \rightleftarrows I_{3} \rightarrow I_{1}$ with $I_{3} \cap I_{2_{1}}=\emptyset$, and by Proposition 2.3, $\operatorname{Per}(f)=\mathbb{N}$. If $I_{3}=\left[b_{0}, p\right]$, denote $b_{1}=\inf \left\{\left(f \mid I_{3}\right)^{-1}\left(a_{0}\right)\right\}$ and $I_{3_{1}}=\left[b_{0}, b_{1}\right]$. Then $f$ has the subgraph $\odot I_{4} \rightarrow I_{3_{1}} \rightleftarrows I_{2} \rightarrow I_{4}$ with $I_{2} \cap I_{3_{1}}=\emptyset$, and by Proposition 2.3, $\operatorname{Per}(f)=\mathbb{N}$.

We consider now $I_{2}=\left[p, a_{0}\right]$. If $I_{3}=\left[p, b_{0}\right]$, set $b_{1}=\sup \left\{\left(f \mid I_{3}\right)^{-1}\left(a_{0}\right)\right\}$ and $I_{3_{2}}=\left[b_{1}, b_{0}\right]$. Then $f$ has the subgraph $\odot I_{4} \rightarrow I_{3_{2}} \rightleftarrows I_{2} \rightarrow I_{4}$ with $I_{2} \cap I_{3_{2}}=\emptyset$, and by Proposition 2.3, $\operatorname{Per}(f)=\mathbb{N}$. If $I_{3}=\left[b_{0}, p\right]$, write $a_{1}=\sup \left\{\left(f \mid I_{2}\right)^{-1}\left(b_{0}\right)\right\}$ and $I_{2_{2}}=\left[a_{1}, a_{0}\right]$. Then $f$ has the subgraph $\odot I_{1} \rightarrow I_{2_{2}} \rightleftarrows I_{3} \rightarrow I_{1}$ with $I_{3} \cap I_{2_{2}}=\emptyset$, and by Proposition 2.3, $\operatorname{Per}(f)=\mathbb{N}$. Therefore, if $b=-1$ and $c=1$, then $\operatorname{MPer}(f)=\mathbb{N}$.

We consider now the case $\mathbf{b}=\mathbf{1}$ and $\mathbf{c}=\mathbf{- 1}$. We know that $f$ has four basic intervals $I_{1}$, $I_{2}, I_{3}$, and $I_{4}$, the first two in $C_{1}$ and the other two in $C_{2}$, such that $f\left(I_{1}\right)=f\left(I_{3}\right)=C_{1}$ and $f\left(I_{2}\right)=f\left(I_{4}\right)=C_{2}$. We have again four possibilities for these intervals. Let $a_{0} \in I_{1} \cap I_{2}$ and $b_{0} \in I_{3} \cap I_{4}$ (see for instance Fig. 14). We take the interval $I_{2}$ to be $\left[a_{0}, p\right]$. If $I_{3}=\left[p, b_{0}\right]$, define $b_{1}=\sup \left\{\left(f \mid I_{3}\right)^{-1}\left(a_{0}\right)\right\}$ and $I_{3_{2}}=\left[b_{1}, b_{0}\right]$. It follows that $f$ has the subgraph $\odot I_{4} \rightarrow$ $I_{3_{2}} \rightleftarrows I_{2} \rightarrow I_{4}$ with $I_{2} \cap I_{3_{2}}=\emptyset$, and by Proposition 2.3, $\operatorname{Per}(f)=\mathbb{N}$. If $I_{3}=\left[b_{0}, p\right]$, consider $a_{1}=\inf \left\{\left(f \mid I_{2}\right)^{-1}\left(b_{0}\right)\right\}$ and $I_{2_{1}}=\left[a_{0}, a_{1}\right]$. Then $f$ has the subgraph $\subset I_{1} \rightarrow I_{2_{1}} \rightleftarrows I_{3} \rightarrow I_{1}$ with $I_{3} \cap I_{2_{1}}=\emptyset$, and we get, by Proposition 2.3, $\operatorname{Per}(f)=\mathbb{N}$.

Suppose that $I_{2}=\left[p, a_{0}\right]$. If $I_{3}=\left[p, b_{0}\right]$, set $a_{1}=\sup \left\{\left(f \mid I_{2}\right)^{-1}\left(b_{0}\right)\right\}$ and $I_{2_{2}}=\left[a_{1}, a_{0}\right]$. Then $f$ has the subgraph $\odot I_{1} \rightarrow I_{2_{2}} \rightleftarrows I_{3} \rightarrow I_{1}$ with $I_{3} \cap I_{2_{2}}=\emptyset$, and by Proposition 2.3, $\operatorname{Per}(f)=\mathbb{N}$. 


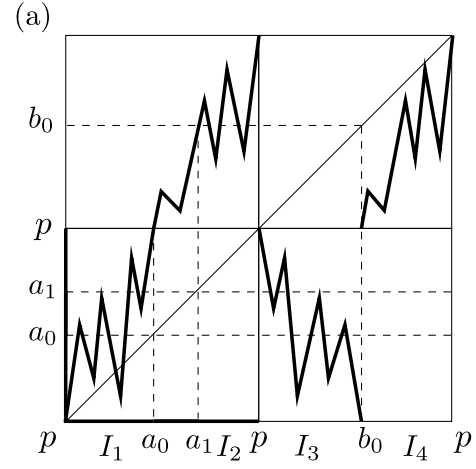

(c)

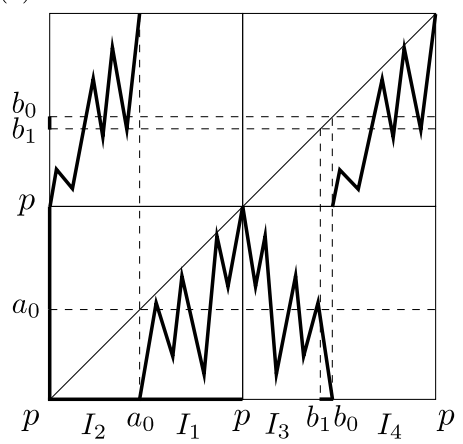

(b)

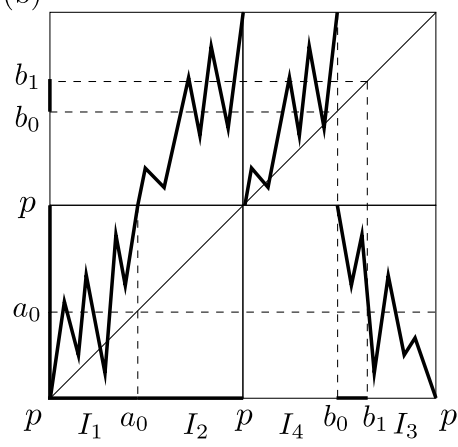

(d)

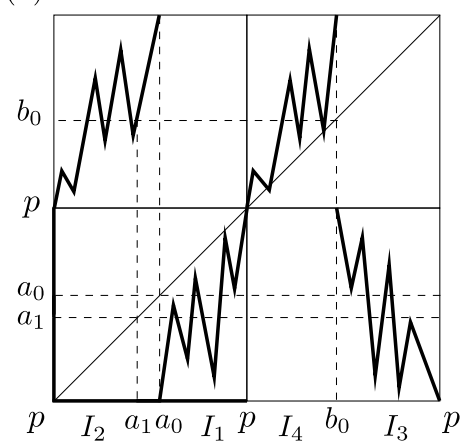

Figure 13 Examples of maps with $\{a, d\} \subset\{-1,0,1\},|a|+|d|=2, b=-1$, and $c=1$

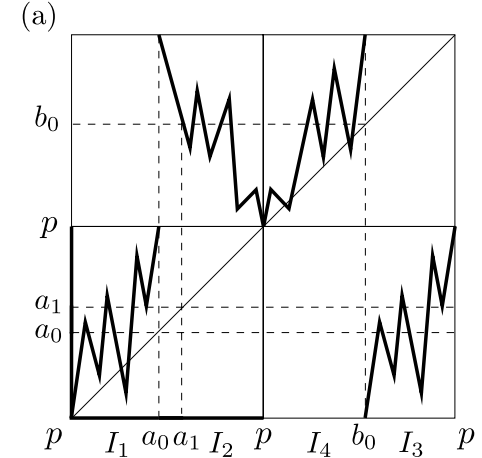

(c)

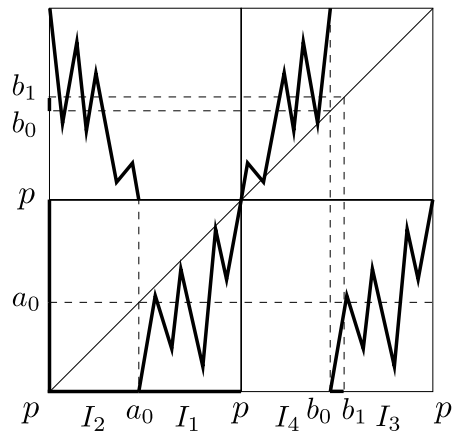

(b)

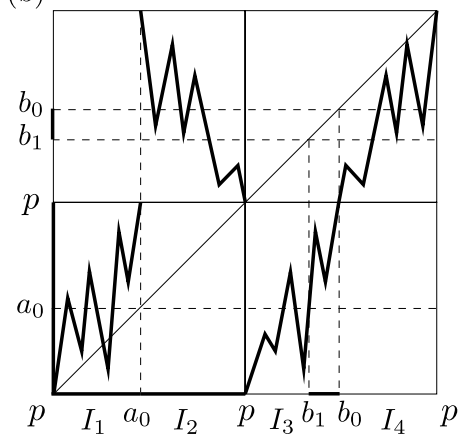

(d)

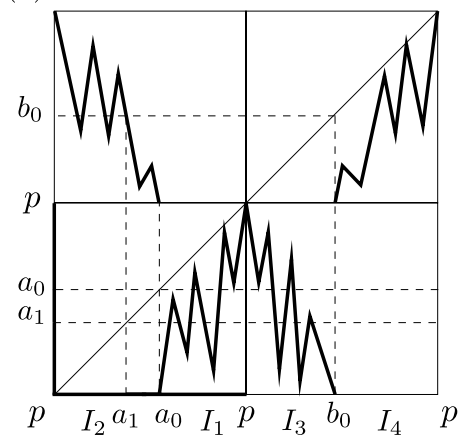

Figure 14 Examples of maps with $\{a, d\} \subset\{-1,0,1\},|a|+|d|=2, b=1$, and $c=-1$ 
If $I_{3}=\left[b_{0}, p\right]$, consider $b_{1}=\inf \left\{\left(f \mid I_{3}\right)^{-1}\left(a_{0}\right)\right\}$ and $I_{3_{1}}=\left[b_{0}, b_{1}\right]$. Then $f$ has the subgraph $\odot I_{4} \rightarrow I_{3_{1}} \rightleftarrows I_{2} \rightarrow I_{4}$ with $I_{2} \cap I_{3_{1}}=\emptyset$, and by Proposition 2.3, $\operatorname{Per}(f)=\mathbb{N}$. Therefore, if $b=1$ and $c=-1$, then $\operatorname{MPer}(f)=\mathbb{N}$. Hence, if $|a|+|d|=2$ and $b c=-1$, then $\operatorname{MPer}(f)=\mathbb{N}$. This completes the proof of statement (c1).

Proof of statement (c21) of Theorem B We assume now that $\mathbf{a}=\mathbf{1}$ and $\mathbf{d}=\mathbf{0}$. If $\mathbf{b c}=\mathbf{0}$, then $\operatorname{MPer}(f)=\{1\}$ as it can be deduced from the examples of Fig. 15. We suppose that $b$ and $c$ are such that $|\mathbf{b c}|>\mathbf{1}$ and $(\mathbf{b}, \mathbf{c}) \notin\{(\mathbf{2}, \mathbf{1}),(\mathbf{2},-\mathbf{1}),(-\mathbf{2}, \mathbf{1}),(-\mathbf{2},-\mathbf{1})\}$. From the graph of $f$ (see for instance Fig. 16) it follows that there are three basic intervals $I_{1}, I_{2}$, and $I_{3}, I_{1}, I_{2} \subset C_{1}$, $I_{3} \subset C_{2}$, such that either $p \notin I_{1} \cap I_{2}$ or $p \notin I_{1} \cap I_{3}$ and $f$ has the subgraph of Proposition 2.3, so $\operatorname{Per}(f) \supset \mathbb{N} \backslash\{2\}$.

If $\{\mathbf{b}, \mathbf{c}\} \not \subset\{-\mathbf{2},-\mathbf{1}, \mathbf{1}, \mathbf{2}\}$, then we can choose $I_{2}$ and $I_{3}$ such that $I_{2} \cap I_{3}=\emptyset$, and by Proposition $2.3,2 \in \operatorname{Per}(f)$. If $\{\mathbf{b}, \mathbf{c}\} \subset\{\mathbf{- 2}, \mathbf{- 1}, \mathbf{1}, \mathbf{2}\}$, in general there do not exist two basic intervals $I_{i}$ and $I_{j}, I_{i} \neq I_{j}$, such that $p \notin I_{i} \cap I_{j}$ and $I_{i} \rightleftarrows I_{j}$. If they exist, then by Lemma 2.1, considering the non-repetitive loop $I_{i} \rightarrow I_{j} \rightarrow I_{i}$, there is a periodic point $z$ of $f$ with period 2 . If they do not exist (see for instance (c) and (d) of Fig. 16) and $(b, c) \in\{(1,2),(-1,-2)\}, 2 \notin \operatorname{Per}(f)$ as we can see from the examples of Fig. 17. Now we will prove that if $(b, c) \in\{(1,-2),(-1,2)\}$ or $|b|=|c|=2$, then $2 \in \operatorname{Per}(f)$.

We suppose first that $(b, c) \in\{(1,-2),(-1,2)\}$. We know that $f$ has four basic intervals $I_{1}$, $I_{2}, I_{3}$, and $I_{4}$, the first three in $C_{1}$ and $I_{4}=C_{2}$, such that $f\left(I_{1}\right)=f\left(I_{4}\right)=C_{1}$ and $f\left(I_{2}\right)=f\left(I_{3}\right)=$ $C_{2}$. Let $p$ and $a_{0}$ be the endpoints of $I_{2}, a_{0}$ and $a_{1}$ be the endpoints of $I_{1}, a_{1}$ and $p$ be the endpoints of $I_{3}$ (see for instance Fig. 18). We consider an ordering in the intervals $I_{1}, I_{2}$, and $I_{3}$ in such a way that $p$ is the smallest element of $I_{2}$ and the greatest of $I_{3}$. Under these

Figure 15 Examples of maps with $a=1, d=0$, and $b c=0$
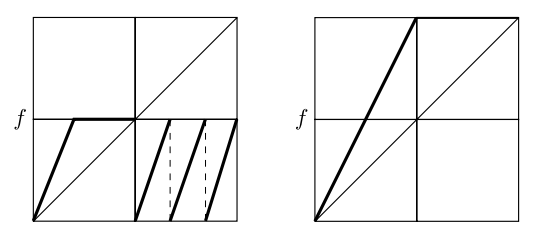

(a)

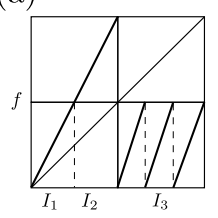

(b)

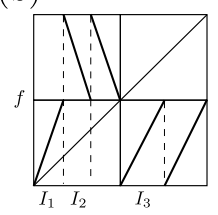

(c)

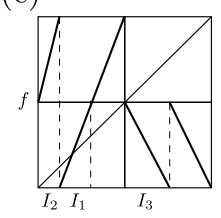

(d)

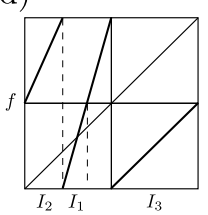

(e)

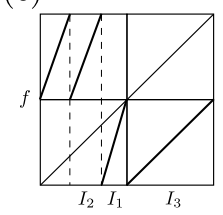

Figure 16 Examples of maps with $a=1, d=0,(b, c) \notin\{(2,1),(2,-1),(-2,1),(-2,-1)\}$, and $|b c|>1$
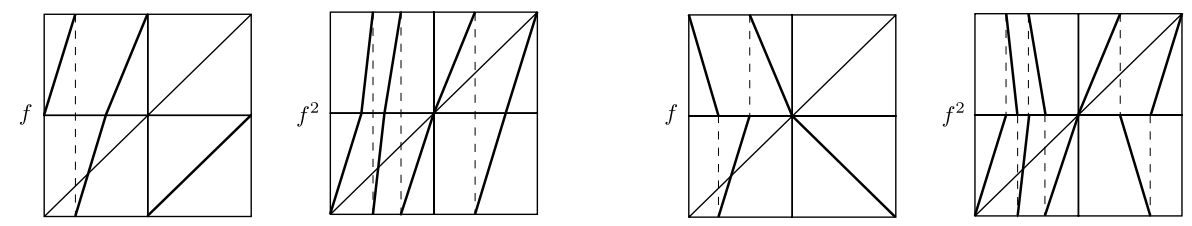

Figure 17 Examples of maps with $a=1, d=0,(b, c) \in\{(1,2),(-1,-2)\}$, and $2 \notin \operatorname{Per}(f)$ 

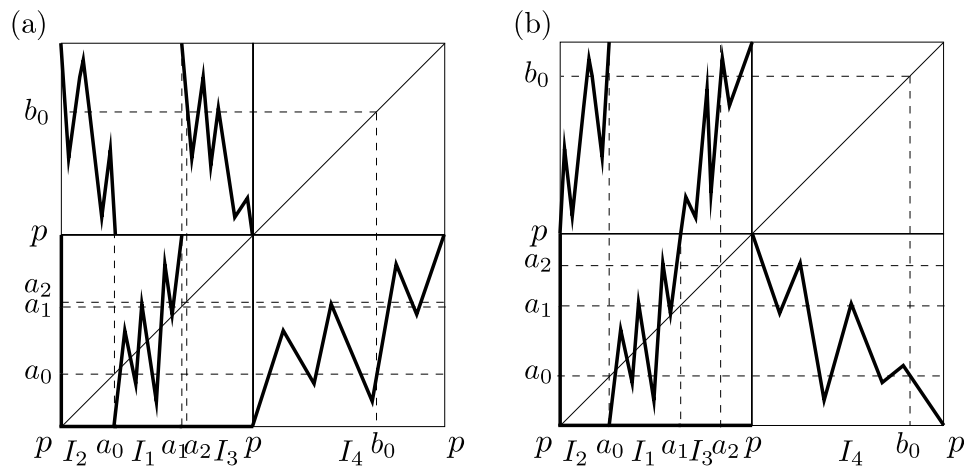

Figure 18 Examples of maps with $a=1, d=0$, and $(b, c) \in\{(1,-2),(-1,2)\}$

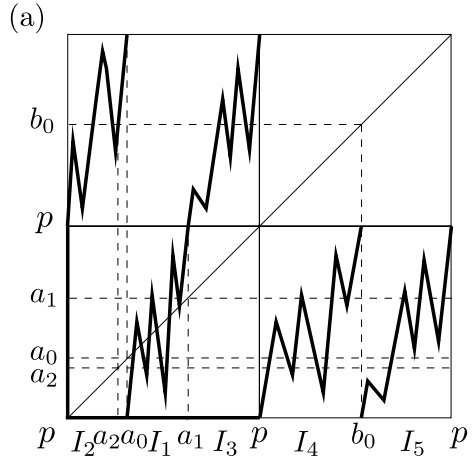

(c)

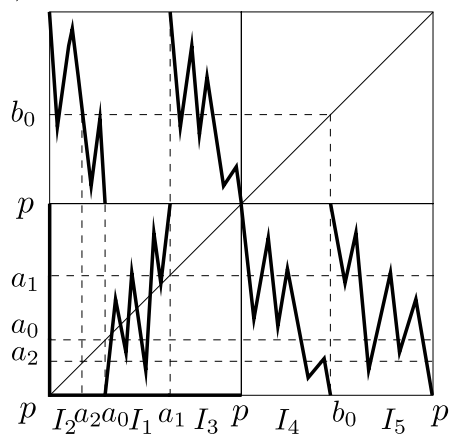

(b)

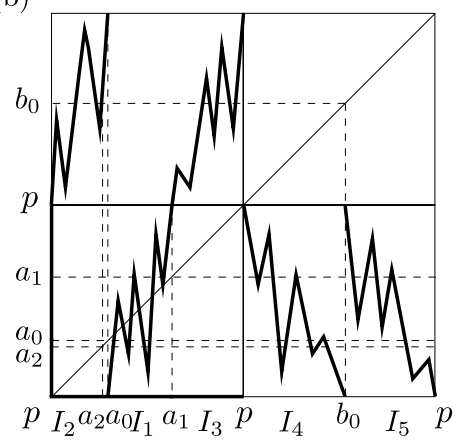

(d)

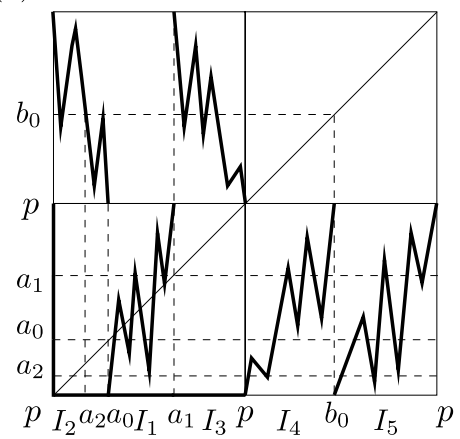

Figure 19 Examples of maps with $a=1, d=0$, and $|b|=|c|=2$

assumptions, set $I_{2}=\left[p, a_{0}\right], I_{1}=\left[a_{0}, a_{1}\right]$, and $I_{3}=\left[a_{1}, p\right]$. Define $b_{0}=\sup \left\{\left(f \mid I_{4}\right)^{-1}\left(a_{0}\right)\right\}, I_{4_{1}}=$ $\left[p, b_{0}\right]$, and $I_{4_{2}}=\left[b_{0}, p\right]$. Set $a_{2}=\inf \left\{\left(f \mid I_{3}\right)^{-1}\left(b_{0}\right)\right\}$ and $I_{3_{1}}=\left[a_{1}, a_{2}\right]$. If $(b, c)=(1,-2)$, we have $I_{4_{2}} \rightleftarrows I_{3_{1}}$ and $I_{4_{2}} \cap I_{3_{1}}=\emptyset$. If $(b, c)=(-1,2)$, we get $I_{4_{1}} \rightleftarrows I_{3_{1}}$ and $I_{4_{1}} \cap I_{3_{1}}=\emptyset$. So, by Lemma 2.1, $2 \in \operatorname{Per}(f)$.

Suppose now that $|b|=|c|=2$. We know that $f$ has five basic intervals $I_{1}, I_{2}, I_{3}, I_{4}$, and $I_{5}$, the first three in $C_{1}$ and the other two in $C_{2}$, such that $f\left(I_{2}\right)=f\left(I_{3}\right)=C_{2}$ and $f\left(I_{1}\right)=$ $f\left(I_{4}\right)=f\left(I_{5}\right)=C_{1}$. Taking an ordering similar to the previous case, define the intervals $I_{2}=$ $\left[p, a_{0}\right], I_{1}=\left[a_{0}, a_{1}\right], I_{3}=\left[a_{1}, p\right], I_{4}=\left[p, b_{0}\right]$, and $I_{5}=\left[b_{0}, p\right]$ (see for instance Fig. 19). Set $a_{2}=\sup \left\{\left(f \mid I_{2}\right)^{-1}\left(b_{0}\right)\right\}$ and $I_{2_{2}}=\left[a_{2}, a_{0}\right]$. If $c=2$, we have $I_{2_{2}} \rightleftarrows I_{5}$ and $I_{2_{2}} \cap I_{5}=\emptyset$. If $c=-2$, we have $I_{2_{2}} \rightleftarrows I_{4}$ and $I_{2_{2}} \cap I_{4}=\emptyset$. So, by Lemma 2.1, $2 \in \operatorname{Per}(f)$. Therefore, if $|b c|>1$ and 


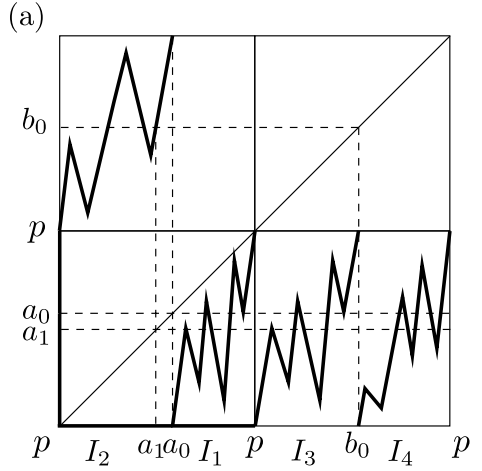

(c)

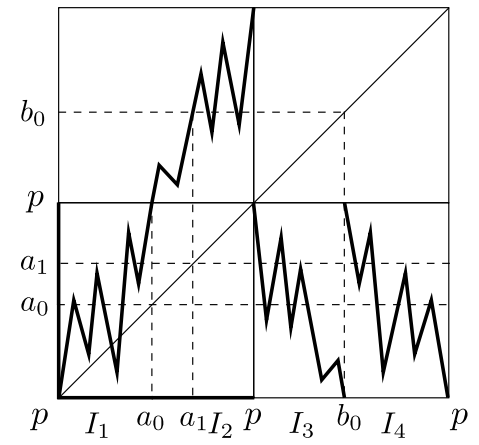

(b)

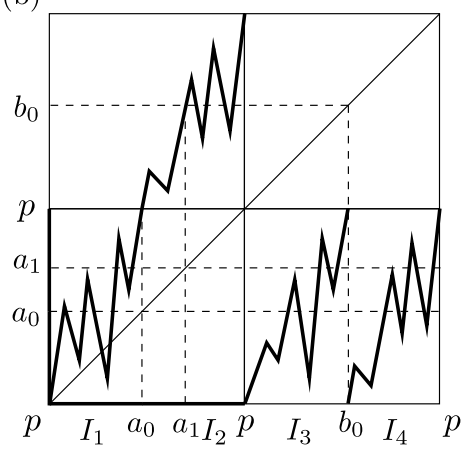

(d)

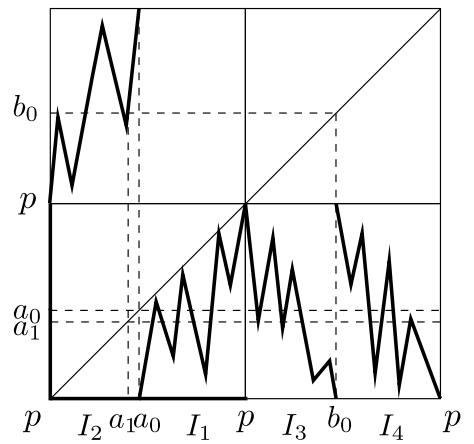

Figure 20 Examples of maps with $a=1, d=0$, and $(b, c) \in\{(2,1),(-2,1)\}$

$(b, c) \notin\{(2,1),(2,-1),(-2,1),(-2,-1)\}$, we have $\operatorname{MPer}(f)=\mathbb{N} \backslash\{2\}$ if $(b, c) \in\{(1,2),(-1,-2)\}$ and $\operatorname{MPer}(f)=\mathbb{N}$ otherwise.

We assume that $|\mathbf{b c}|>\mathbf{1}$ and $(\mathbf{b}, \mathbf{c}) \in\{(\mathbf{2}, \mathbf{1}),(\mathbf{2},-\mathbf{1}),(-\mathbf{2}, \mathbf{1}),(-\mathbf{2},-\mathbf{1})\}$. We know that $f$ has four basic intervals $I_{1}, I_{2}, I_{3}$, and $I_{4}$, the first two in $C_{1}$ and the others in $C_{2}$, such that $f\left(I_{1}\right)=$ $f\left(I_{3}\right)=f\left(I_{4}\right)=C_{1}$ and $f\left(I_{2}\right)=C_{2}$. Let $p$ and $a_{0}$ be the endpoints of $I_{1}$ and $I_{2}$, and $b_{0}$ and $p$ be the endpoints of $I_{3}$ and $I_{4}$ (see for instance Figs. 20 and 21). For each pair $(b, c)$, we have two possibilities for the intervals $I_{1}$ and $I_{2}$. If $(b, c) \in\{(2,1),(-2,1)\}$ and $I_{2}=\left[a_{0}, p\right]$, write $a_{1}=\inf \left\{\left(f \mid I_{2}\right)^{-1}\left(b_{0}\right)\right\}$ and $I_{2_{1}}=\left[a_{0}, a_{1}\right]$. Then $f$ has the subgraph $\odot I_{1} \rightarrow I_{2_{1}} \rightleftarrows I_{3} \rightarrow I_{1}$ with $I_{3} \cap I_{2_{1}}=\emptyset$, and by Proposition 2.3, $\operatorname{Per}(f)=\mathbb{N}$. If $I_{2}=\left[p, a_{0}\right]$, consider $a_{1}=\sup \left\{\left(f \mid I_{2}\right)^{-1}\left(b_{0}\right)\right\}$ and $I_{2_{2}}=\left[a_{1}, a_{0}\right]$. Then $f$ has the subgraph $\odot I_{1} \rightarrow I_{2_{2}} \rightleftarrows I_{4} \rightarrow I_{1}$ with $I_{4} \cap I_{2_{2}}=\emptyset$, and by Proposition 2.3, $\operatorname{Per}(f)=\mathbb{N}$.

If $(b, c) \in\{(-2,-1),(2,-1)\}$ and $I_{2}=\left[a_{0}, p\right]$, set $a_{1}=\inf \left\{\left(f \mid I_{2}\right)^{-1}\left(b_{0}\right)\right\}$ and $I_{2_{1}}=\left[a_{0}, a_{1}\right]$. Then $f$ has the subgraph $\odot I_{1} \rightarrow I_{2_{1}} \rightleftarrows I_{4} \rightarrow I_{1}$ with $I_{4} \cap I_{2_{1}}=\emptyset$, and by Proposition 2.3, $\operatorname{Per}(f)=\mathbb{N}$. If $I_{2}=\left[p, a_{0}\right]$, consider $a_{1}=\sup \left\{\left(f \mid I_{2}\right)^{-1}\left(b_{0}\right)\right\}$ and $I_{2_{2}}=\left[a_{1}, a_{0}\right]$. Then $f$ has the subgraph $\odot I_{1} \rightarrow I_{2_{2}} \rightleftarrows I_{3} \rightarrow I_{1}$ with $I_{3} \cap I_{2_{2}}=\emptyset$, and by Proposition 2.3, $\operatorname{Per}(f)=\mathbb{N}$. Therefore, if $|\mathbf{b c}|>\mathbf{1}$ and $(\mathbf{b}, \mathbf{c}) \in\{(\mathbf{2}, \mathbf{1}),(\mathbf{2},-\mathbf{1}),(-\mathbf{2}, \mathbf{1}),(-\mathbf{2},-\mathbf{1})\}, \operatorname{MPer}(f)=\mathbb{N}$.

We consider the case $|\mathbf{b c}|=\mathbf{1}$. First assume that $\mathbf{b c}=\mathbf{1}$. As we can see from the examples of Fig. 22, $2 \notin \operatorname{MPer}(f)$. Now we will prove that $\operatorname{Per}(f)=\mathbb{N} \backslash\{2\}$.

We know that $f$ has three basic intervals $I_{1}, I_{2}$, and $I_{3}$, the first two in $C_{1}$ and $I_{3}=C_{2}$, such that $f\left(I_{1}\right)=f\left(I_{3}\right)=C_{1}$ and $f\left(I_{2}\right)=C_{2}$. We have two possibilities for the intervals $I_{1}$ and $I_{2}$ : either $p$ is the smallest element of $I_{1}$ and the greatest of $I_{2}$ or $p$ is the smallest element of $I_{2}$ and the greatest of $I_{1}$ (see for instance Fig. 23). In the assumption that $b=$ $c=1$, if $I_{1}=\left[p, a_{0}\right]$, write $b_{0}=\inf \left\{\left(f \mid I_{3}\right)^{-1}\left(a_{0}\right)\right\}, I_{3_{1}}=\left[p, b_{0}\right], a_{1}=\inf \left\{\left(f \mid I_{2}\right)^{-1}\left(b_{0}\right)\right\}$, and $I_{2_{1}}=$ 
(a)

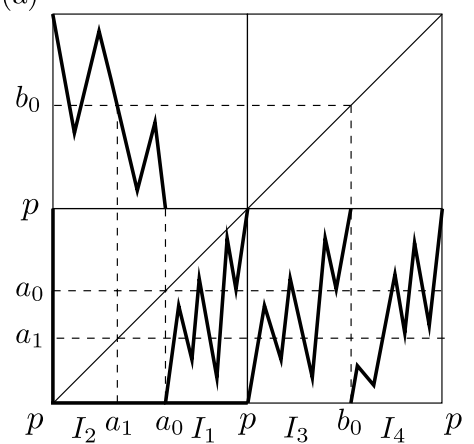

(c)

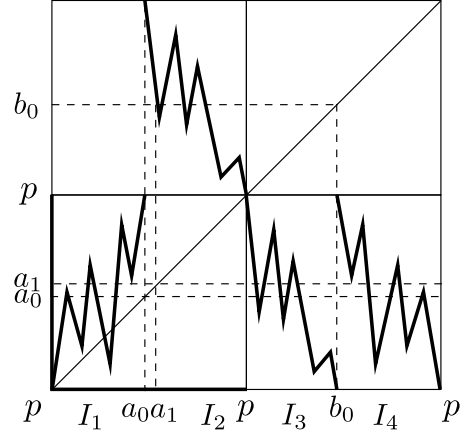

(b)

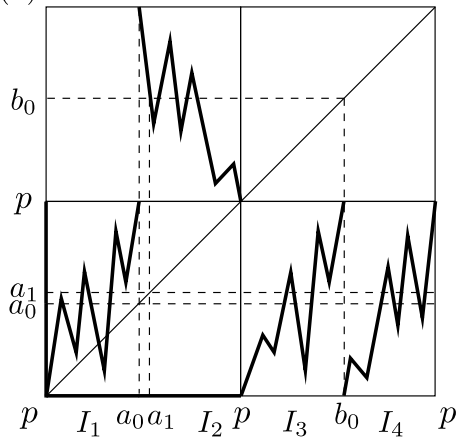

(d)

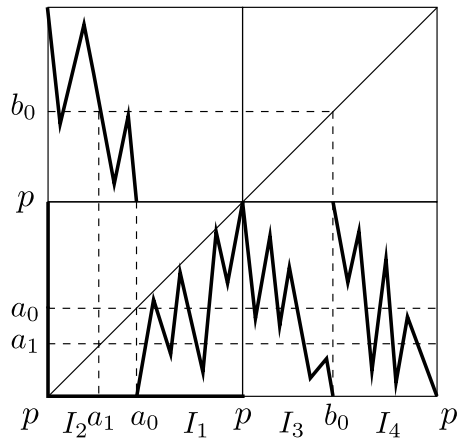

Figure 21 Examples of maps with $a=1, d=0$, and $(b, c) \in\{(-2,-1),(2,-1)\}$
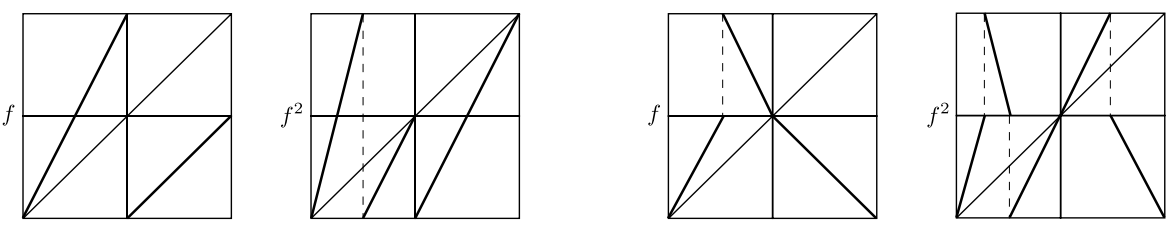

Figure 22 Examples of maps with $a=1, d=0, b c=1$, and $2 \notin \operatorname{Per}(f)$

$\left[a_{0}, a_{1}\right]$. Then $f$ has the subgraph $\odot I_{1} \rightarrow I_{2_{1}} \rightarrow I_{3_{1}} \rightarrow I_{1}$ and by Proposition 2.3, $\operatorname{Per}(f) \supset$ $\mathbb{N} \backslash\{2\}$. If $I_{1}=\left[a_{0}, p\right]$, define $b_{0}=\sup \left\{\left(f \mid I_{3}\right)^{-1}\left(a_{0}\right)\right\}, I_{3_{2}}=\left[b_{0}, p\right], a_{1}=\sup \left\{\left(f \mid I_{2}\right)^{-1}\left(b_{0}\right)\right\}$, and $I_{2_{2}}=\left[a_{1}, a_{0}\right]$. Then $f$ has the subgraph $\odot I_{1} \rightarrow I_{2_{2}} \rightarrow I_{3_{2}} \rightarrow I_{1}$, and by Proposition 2.3, $\operatorname{Per}(f) \supset \mathbb{N} \backslash\{2\}$.

If $b=c=-1$, we consider first the case $I_{1}=\left[p, a_{0}\right]$. Set $b_{0}=\sup \left\{\left(f \mid I_{3}\right)^{-1}\left(a_{0}\right)\right\}, I_{3_{2}}=\left[b_{0}, p\right]$, $a_{1}=\inf \left\{\left(f \mid I_{2}\right)^{-1}\left(b_{0}\right)\right\}$, and $I_{2_{1}}=\left[a_{0}, a_{1}\right]$. Then $f$ has the subgraph $\odot I_{1} \rightarrow I_{2_{1}} \rightarrow I_{3_{2}} \rightarrow I_{1}$, and by Proposition 2.3, $\operatorname{Per}(f) \supset \mathbb{N} \backslash\{2\}$. If $I_{1}=\left[a_{0}, p\right]$, write $b_{0}=\inf \left\{\left(f \mid I_{3}\right)^{-1}\left(a_{0}\right)\right\}, I_{3_{1}}=$ $\left[p, b_{0}\right], a_{1}=\sup \left\{\left(f \mid I_{2}\right)^{-1}\left(b_{0}\right)\right\}$, and $I_{2_{2}}=\left[a_{1}, a_{0}\right]$. Then $f$ has the subgraph $\odot I_{1} \rightarrow I_{2_{2}} \rightarrow$ $I_{3_{1}} \rightarrow I_{1}$, and by Proposition 2.3, $\operatorname{Per}(f) \supset \mathbb{N} \backslash\{2\}$. Therefore, if $a=1, d=0$, and $b c=1$, $\operatorname{MPer}(f)=\mathbb{N} \backslash\{2\}$.

Assume now that $\mathbf{b c}=-\mathbf{1}$. We know that $f$ has three basic intervals $I_{1}, I_{2}$, and $I_{3}$, the first two in $C_{1}$ and $I_{3}=C_{2}$, such that $f\left(I_{1}\right)=f\left(I_{3}\right)=C_{1}$ and $f\left(I_{2}\right)=C_{2}$. We have two possibilities for the intervals $I_{1}$ and $I_{2}$ : either $p$ is the smallest element of $I_{1}$ and the greatest of $I_{2}$ or $p$ is the smallest element of $I_{2}$ and the greatest of $I_{1}$ (see for instance Fig. 24). Define $b_{0}=$ $\inf \left\{\left(f \mid I_{3}\right)^{-1}\left(a_{0}\right)\right\}, I_{3_{1}}=\left[p, b_{0}\right], I_{3_{2}}=\left[b_{0}, p\right]$, and $a_{1}=\inf \left\{\left(f \mid I_{2}\right)^{-1}\left(b_{0}\right)\right\}$. 
(a)

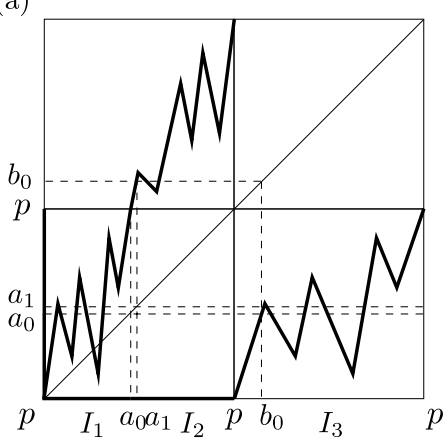

(c)

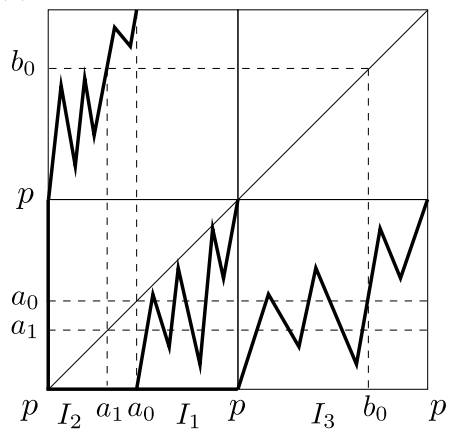

(b)

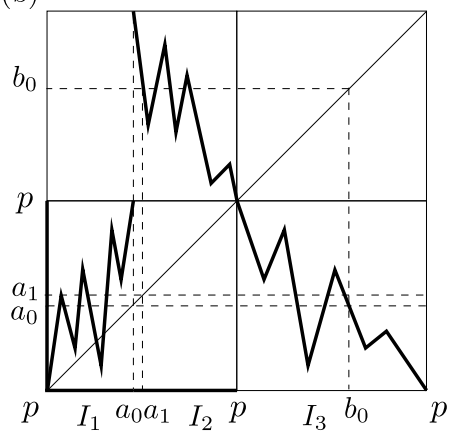

(d)

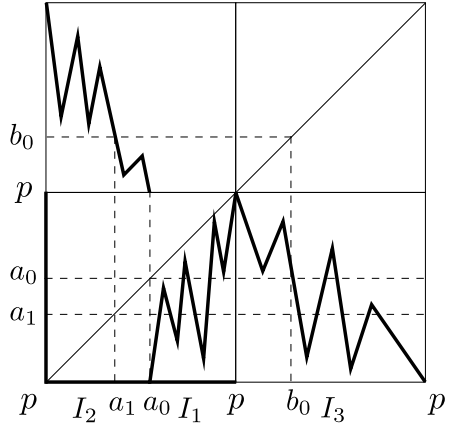

Figure 23 Examples of maps with $a=1, d=0$, and $b c=1$

(a)

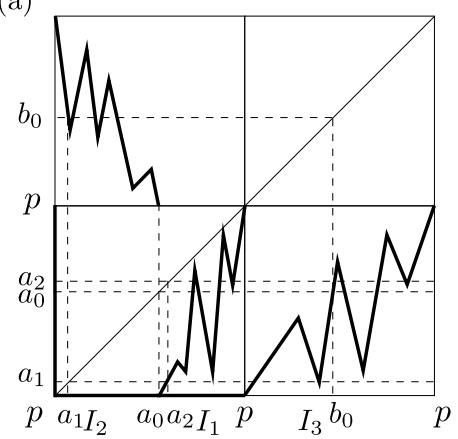

(c)

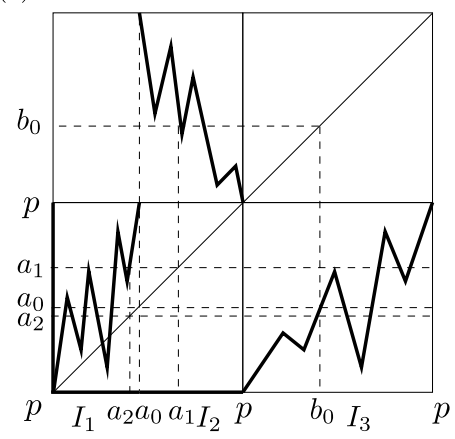

(b)

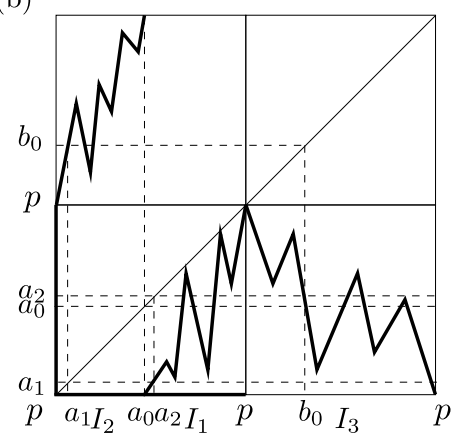

(d)

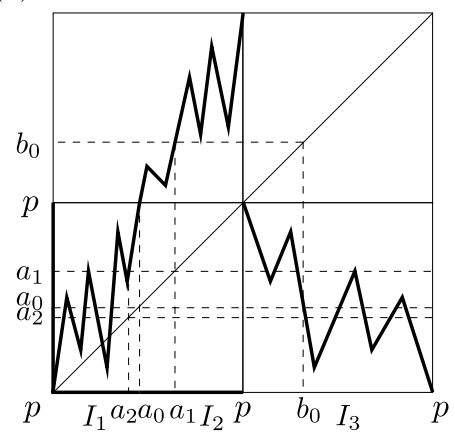

Figure 24 Examples of maps with $a=1, d=0$, and $b c=-1$ 
If $I_{1}=\left[a_{0}, p\right]$, let $I_{2_{1}}=\left[p, a_{1}\right]$ and $I_{2_{2}}=\left[a_{1}, a_{0}\right]$. Consider $a_{2}=\inf \left\{\left(f \mid I_{1}\right)^{-1}\left(a_{1}\right)\right\}$. We write $I_{1_{1}}=\left[a_{0}, a_{2}\right]$ and $I_{1_{2}}=\left[a_{2}, p\right]$. If $b=1$ and $c=-1$ (see (a) of Fig. 24), $f$ has the subgraph

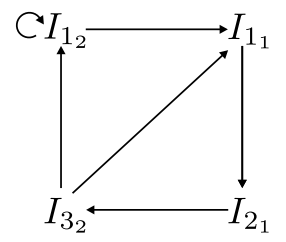

We consider the non-repetitive loops $I_{1_{1}} \rightarrow I_{2_{1}} \rightarrow I_{3_{2}} \rightarrow I_{1_{1}}$ and $I_{1_{2}} \rightarrow I_{1_{1}} \rightarrow I_{2_{1}} \rightarrow I_{3_{2}} \rightarrow$ $I_{1_{2}} \rightarrow \cdots \rightarrow I_{1_{2}}$ of lengths 3 and $n \geq 4$, respectively. From the first loop and by Lemma 2.1, there is a periodic point $z$ of $f$ with period 3; from the second loop and by Lemma 2.1, there is a periodic point $z$ of $f$ with period $n \geq 4$. Moreover, $I_{3_{1}} \rightleftarrows I_{2_{2}}$ and $I_{3_{1}} \cap I_{2_{2}}=\emptyset$, so, by Lemma 2.1, $2 \in \operatorname{Per}(f)$. Hence, $\operatorname{Per}(f)=\mathbb{N}$. If $b=-1$ and $c=1$ (see (b) of Fig. 24), $f$ has the subgraph

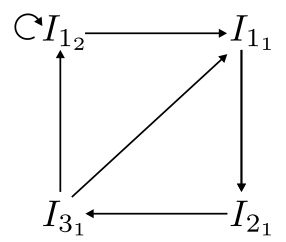

Now from the non-repetitive loops $I_{1_{1}} \rightarrow I_{2_{1}} \rightarrow I_{3_{1}} \rightarrow I_{1_{1}}$ and $I_{1_{2}} \rightarrow I_{1_{1}} \rightarrow I_{2_{1}} \rightarrow I_{3_{1}} \rightarrow$ $I_{1_{2}} \rightarrow \cdots \rightarrow I_{1_{2}}$ of lengths 3 and $n \geq 4$, respectively, and $I_{3_{2}} \rightleftarrows I_{2_{2}}$ and $I_{3_{2}} \cap I_{2_{2}}=\emptyset$, it follows that $\operatorname{Per}(f)=\mathbb{N}$.

If $I_{1}=\left[p, a_{0}\right]$, let $I_{2_{1}}=\left[a_{0}, a_{1}\right], I_{2_{2}}=\left[a_{1}, p\right]$. Define $a_{2}=\sup \left\{\left(f \mid I_{1}\right)^{-1}\left(a_{1}\right)\right\}, I_{1_{1}}=\left[a_{0}, a_{2}\right]$, and $I_{1_{2}}=\left[a_{2}, p\right]$. If $b=1$ and $c=-1$ (see (c) of Fig. 24), $f$ has the subgraph

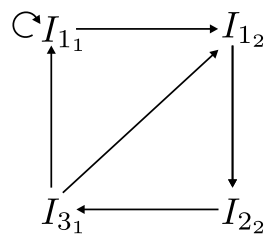

Again from the non-repetitive loops $I_{1_{2}} \rightarrow I_{2_{2}} \rightarrow I_{3_{1}} \rightarrow I_{1_{2}}$ and $I_{1_{1}} \rightarrow I_{1_{2}} \rightarrow I_{2_{2}} \rightarrow I_{3_{1}} \rightarrow$ $I_{1_{1}} \rightarrow \cdots \rightarrow I_{1_{1}}$ of lengths 3 and $n \geq 4$, respectively, $I_{3_{2}} \rightleftarrows I_{2_{1}}$ and $I_{3_{2}} \cap I_{2_{1}}=\emptyset, \operatorname{Per}(f)=\mathbb{N}$. If $b=-1$ and $c=1$ (see (d) of Fig. 24), $f$ has the subgraph

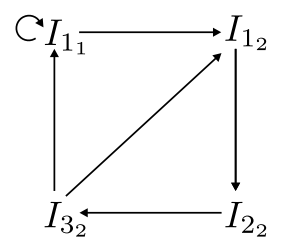

We consider the non-repetitive loops $I_{1_{2}} \rightarrow I_{2_{2}} \rightarrow I_{3_{2}} \rightarrow I_{1_{2}}$ and $I_{1_{1}} \rightarrow I_{1_{2}} \rightarrow I_{2_{2}} \rightarrow I_{3_{2}} \rightarrow$ $I_{1_{1}} \rightarrow \cdots \rightarrow I_{1_{1}}$ of lengths 3 and $n \geq 4$, respectively, $I_{3_{1}} \rightleftarrows I_{2_{1}}$ and $I_{3_{1}} \cap I_{2_{1}}=\emptyset$. We obtain 
that $\operatorname{Per}(f)=\mathbb{N}$. Therefore, if $a=1, d=0$ and $b c=-1, \operatorname{MPer}(f)=\mathbb{N}$. This completes the proof of statement (c21).

Proof of statement (c22) of Theorem B If $\mathbf{a}=\mathbf{0}$ and $\mathbf{d}=\mathbf{1}$, by using the same kind of arguments as those in the case $a=1$ and $d=0$, and interchanging $b$ and $c$, we obtain statement (c22).

Proof of statement (c23) of Theorem B We suppose that $\mathbf{a}=\mathbf{- 1}$ and $\mathbf{d}=\mathbf{0}$. If $\mathbf{b c}=\mathbf{0}$, then $\operatorname{MPer}(f)=\{1\}$ as it can be seen from the examples of Fig. 25. The cases in which $\operatorname{MPer}(f)$ is either $\mathbb{N} \backslash\{2\}$ or $\mathbb{N}$ can be proved following exactly the same kind of arguments as those in the proof of statement (c21).

Assume now that bc $=-\mathbf{1}$. From the examples of Fig. 26 we can see that $3 \notin \operatorname{MPer}(f)$.

We know that $f$ has three basic intervals $I_{1}, I_{2}$, and $I_{3}$, the first two in $C_{1}$ and $I_{3}=C_{2}$, such that $f\left(I_{1}\right)=f\left(I_{3}\right)=C_{1}$ and $f\left(I_{2}\right)=C_{2}$. We have two possibilities for the intervals $I_{1}$ and $I_{2}$ : either $p$ is the smallest element of $I_{1}$ and the greatest of $I_{2}$ or $p$ is the smallest element of $I_{2}$ and the greatest of $I_{1}$ (see for instance Fig. 27). Denote $b_{0}=\inf \left\{\left(f \mid I_{3}\right)^{-1}\left(a_{0}\right)\right\}, I_{3_{1}}=\left[p, b_{0}\right]$ $I_{3_{2}}=\left[b_{0}, p\right]$, and $a_{1}=\inf \left\{\left(f \mid I_{2}\right)^{-1}\left(b_{0}\right)\right\}$.

If $I_{1}=\left[a_{0}, p\right]$, let $I_{2_{1}}=\left[p, a_{1}\right]$ and $I_{2_{2}}=\left[a_{1}, a_{0}\right]$. Consider $a_{2}=\inf \left\{\left(f \mid I_{1}\right)^{-1}\left(a_{1}\right)\right\}$. Write $I_{1_{1}}=\left[a_{0}, a_{2}\right]$ and $I_{1_{2}}=\left[a_{2}, p\right]$. If $b=1$ and $c=-1$ (see (a) of Fig. 27), $f$ has the subgraph $\curvearrowright I_{1_{1}} \rightarrow I_{1_{2}} \rightarrow I_{2_{1}} \rightarrow I_{3_{2}} \rightarrow I_{1_{1}}$. We consider the non-repetitive loop $I_{1_{1}} \rightarrow I_{1_{2}} \rightarrow I_{2_{1}} \rightarrow$ $I_{3_{2}} \rightarrow I_{1_{1}} \rightarrow \cdots \rightarrow I_{1_{1}}$ of length $n \geq 4$. By Lemma 2.1 there is a periodic point $z$ of $f$ with period $n \geq 4$. Moreover, $I_{3_{1}} \rightleftarrows I_{2_{2}}$ and $I_{3_{1}} \cap I_{2_{2}}=\emptyset$, so, by Lemma 2.1, $2 \in \operatorname{Per}(f)$. Hence, $\operatorname{Per}(f)=\mathbb{N} \backslash\{3\}$. If $b=-1$ and $c=1$ (see (b) of Fig. 27), $f$ has the subgraph $\curvearrowright I_{1_{1}} \rightarrow I_{1_{2}} \rightarrow I_{2_{1}} \rightarrow I_{3_{1}} \rightarrow I_{1_{1}}$. We consider the non-repetitive loop $I_{1_{1}} \rightarrow I_{1_{2}} \rightarrow I_{2_{1}} \rightarrow$ $I_{3_{1}} \rightarrow I_{1_{1}} \rightarrow \cdots \rightarrow I_{1_{1}}$ of length $n \geq 4$. By Lemma 2.1 there is a periodic point $z$ of $f$ with

Figure 25 Examples of maps with $a=-1, d=0$, and $b c=0$
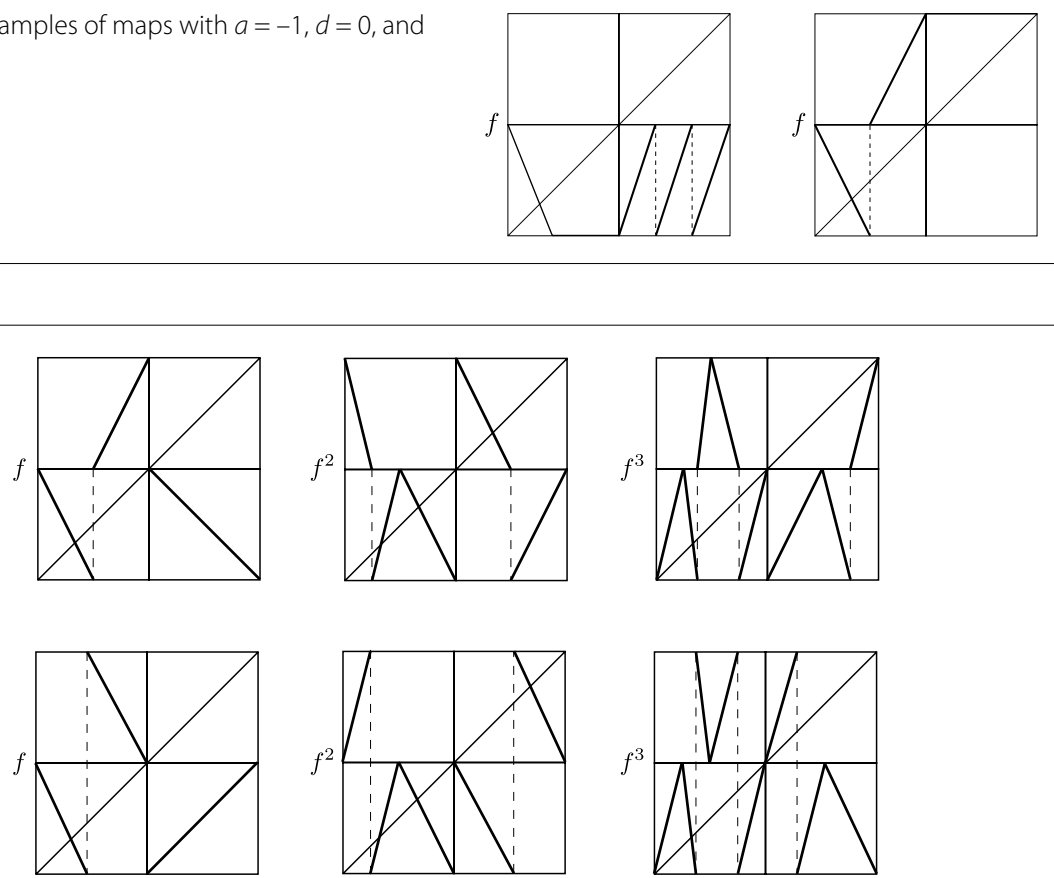

Figure 26 Examples of maps with $a=-1, d=0, b c=-1$, and $3 \notin \operatorname{Per}(f)$ 
(a)

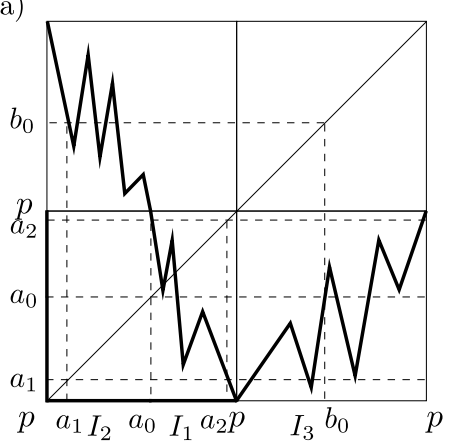

(c)

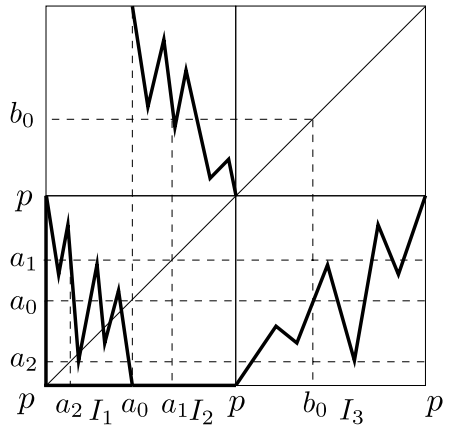

(b)

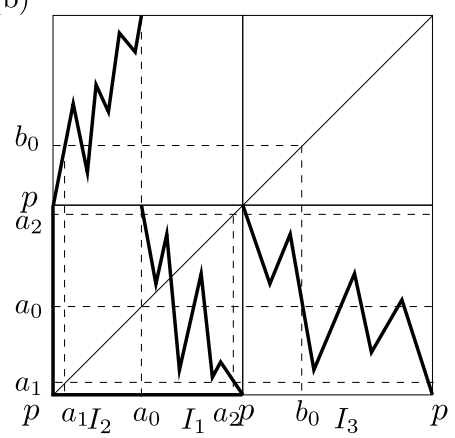

(d)

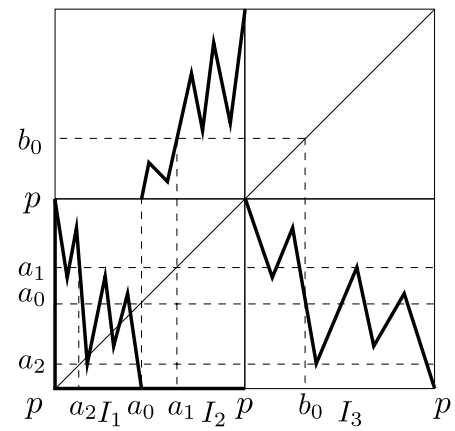

Figure 27 Examples of maps with $a=1, d=0$ and $b c=-1$

period $n \geq 4$. Moreover, $I_{3_{2}} \rightleftarrows I_{2_{2}}$ and $I_{3_{2}} \cap I_{2_{2}}=\emptyset$, so, by Lemma 2.1, $2 \in \operatorname{Per}(f)$. Hence, $\operatorname{Per}(f)=\mathbb{N} \backslash\{3\}$.

If $I_{1}=\left[p, a_{0}\right]$, let $I_{2_{1}}=\left[a_{0}, a_{1}\right]$ and $I_{2_{2}}=\left[a_{1}, p\right]$. Consider $a_{2}=\sup \left\{\left(f \mid I_{1}\right)^{-1}\left(a_{1}\right)\right\}$. Write $I_{1_{1}}=\left[p, a_{2}\right]$ and $I_{1_{2}}=\left[a_{2}, a_{0}\right]$. If $b=1$ and $c=-1$ (see (c) of Fig. 27), $f$ has the subgraph $\propto I_{1_{2}} \rightarrow I_{1_{1}} \rightarrow I_{2_{2}} \rightarrow I_{3_{1}} \rightarrow I_{1_{2}}$. From the non-repetitive loop $I_{1_{2}} \rightarrow I_{1_{1}} \rightarrow I_{2_{2}} \rightarrow I_{3_{1}} \rightarrow$ $I_{1_{2}} \rightarrow \cdots \rightarrow I_{1_{2}}$ of length $n \geq 4, I_{3_{2}} \rightleftarrows I_{2_{1}}$, and $I_{3_{2}} \cap I_{2_{1}}=\emptyset$, we obtain that $\operatorname{Per}(f)=\mathbb{N} \backslash\{3\}$. If $b=-1$ and $c=1$ (see (d) of Fig. 27), $f$ has the subgraph $\odot I_{1_{2}} \rightarrow I_{1_{1}} \rightarrow I_{2_{2}} \rightarrow I_{3_{2}} \rightarrow I_{1_{2}}$. Using the non-repetitive loop $I_{1_{2}} \rightarrow I_{1_{1}} \rightarrow I_{2_{2}} \rightarrow I_{3_{2}} \rightarrow I_{1_{2}} \rightarrow \cdots \rightarrow I_{1_{2}}$ of length $n \geq 4$, $I_{3_{1}} \rightleftarrows I_{2_{1}}$ and $I_{3_{1}} \cap I_{2_{1}}=\emptyset$, we get that $\operatorname{Per}(f)=\mathbb{N} \backslash\{3\}$. Therefore, if $a=-1, d=0$, and $b c=-1, \operatorname{MPer}(f)=\mathbb{N} \backslash\{3\}$. This completes the proof of statement (c23).

Proof of statement (c24) of Theorem B If $\mathbf{a}=\mathbf{0}$ and $\mathbf{d}=-\mathbf{1}$, by using the same kind of arguments as those in the case $a=-1$ and $d=0$, and interchanging $b$ and $c$, we obtain statement (c24).

Proof of statement (c3) of Theorem B We suppose that $\mathbf{a}=\mathbf{d}=\mathbf{0}$. If $\mathbf{b c}=\mathbf{0}$ or $\mathbf{b c}=\mathbf{1}$, we can deduce from the examples of Fig. 28 that $\operatorname{MPer}(f)=\{1\}$. If $\mathbf{b c}=-\mathbf{1}$, then $\operatorname{MPer}(f)=\{1,2\}$ (see for instance Fig. 29).

We assume now that $|\mathbf{b c}|=2$. Since $a=d=0$, we may assume without loss of generality that $|b|=1$ and $|c|=2$. We consider first the case $\mathbf{b c}=\mathbf{- 2}$. Clearly, $\{1,2\} \subset \operatorname{Per}(f)$, no other odd number belongs to $\operatorname{MPer}(f)$ and $4 \notin \operatorname{MPer}(f)$ as it can be deduced from Fig. 30. Now we will prove that $n \in \operatorname{Per}(f)$ for any $n$ even larger than 4 .

We know that $f$ has three basic intervals $I_{1}, I_{2}$, and $I_{3}$, the first two in $C_{1}$ and $I_{3}=C_{2}$, such that $f\left(I_{1}\right)=f\left(I_{2}\right)=C_{2}$ and $f\left(I_{3}\right)=C_{1}$ (see for instance Fig. 31). Consider 

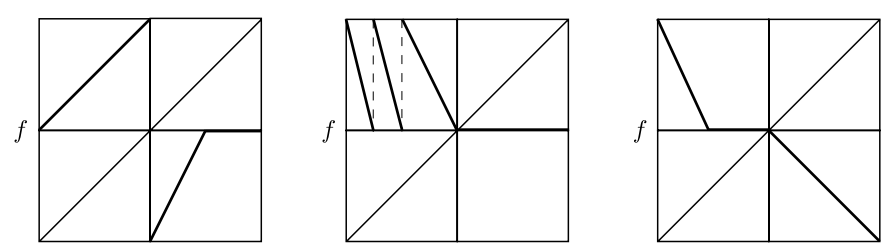

Figure 28 Examples of maps with $a=d=0$ and either $b c=0$ or $b c=1$
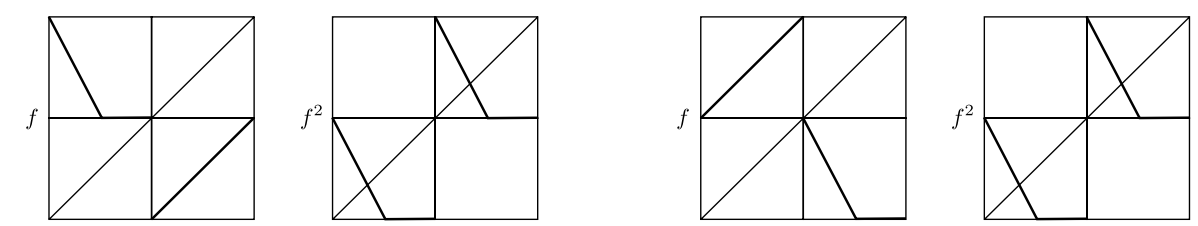

Figure 29 Examples of maps with $a=d=0$ and $b c=-1$
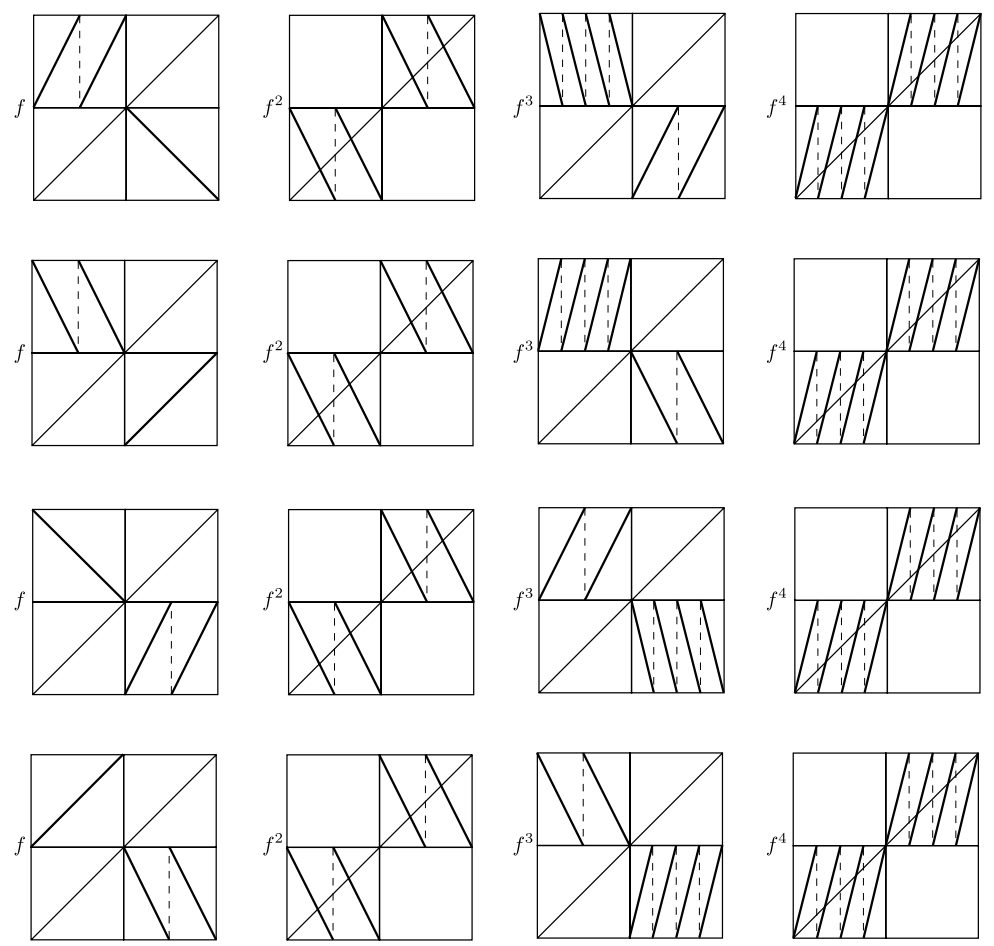

Figure 30 Examples of maps with $a=d=0, b c=-2$, and $4 \notin \operatorname{Per}(f)$ 

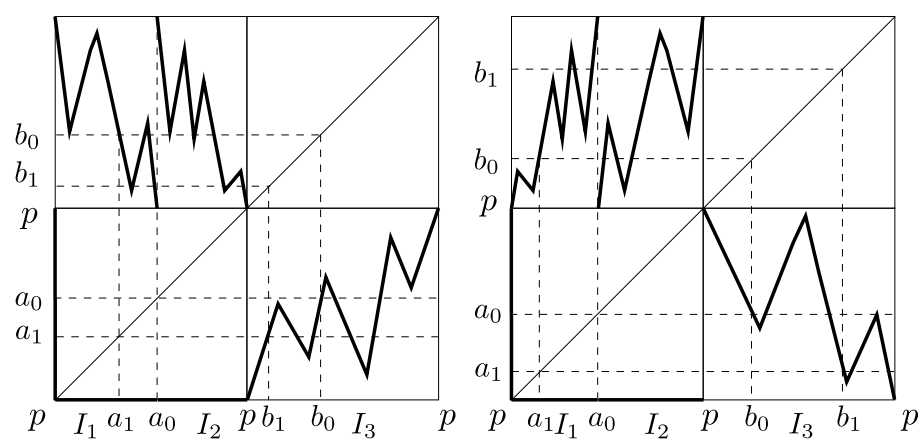

Figure 31 Examples of maps with $a=d=0$ and $b c=-2$
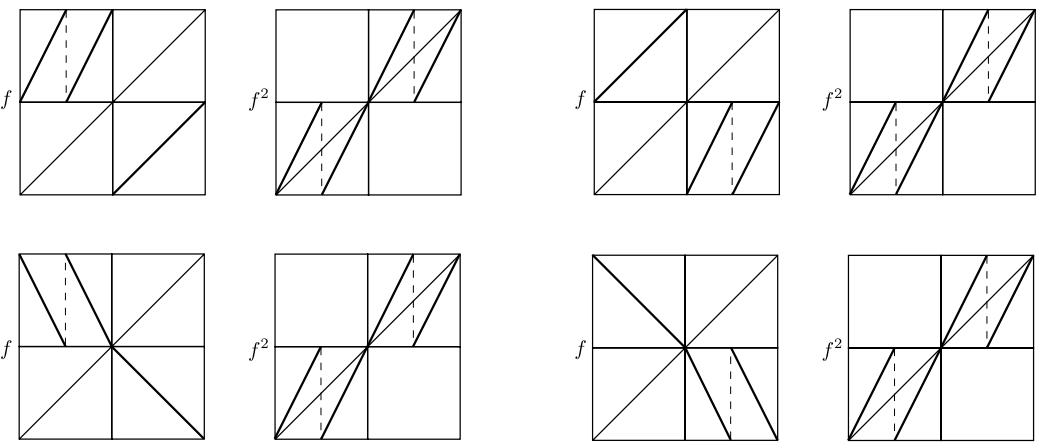

Figure 32 Examples of maps with $a=d=0, b c=2$, and $2 \notin \operatorname{Per}(f)$

$b_{0}=\inf \left\{\left(f \mid I_{3}\right)^{-1}\left(a_{0}\right)\right\}, a_{1}=\inf \left\{\left(f \mid I_{1}\right)^{-1}\left(b_{0}\right)\right\}, b_{1}=\inf \left\{\left(f \mid I_{3}\right)^{-1}\left(a_{1}\right)\right\}$. Set $I_{1_{1}}=\left[p, a_{1}\right], I_{1_{2}}=$ $\left[a_{1}, a_{0}\right], I_{3_{1}}$ the interval with endpoints $b_{1}$ and $p, I_{3_{2}}$ the interval with endpoints $b_{1}$ and $b_{0}$, and $I_{3_{3}}$ the interval with endpoints $b_{0}$ and $p$. Then $f$ has the subgraph

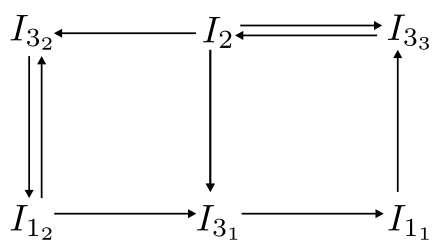

We consider the non-repetitive loops $I_{3_{2}} \rightarrow I_{1_{2}} \rightarrow I_{3_{2}}$ and $I_{2} \rightarrow I_{3_{2}} \rightarrow I_{1_{2}} \rightarrow I_{3_{1}} \rightarrow I_{1_{1}} \rightarrow$ $I_{3_{3}} \rightarrow I_{2} \rightarrow \cdots \rightarrow I_{3_{3}} \rightarrow I_{2}$ of lengths 2 and $n$ even, $n \geq 6$, respectively. We have $I_{3_{2}} \cap I_{1_{2}}=$ $\emptyset$, so, from the first loop and by Lemma 2.1, there is a periodic point $z$ of $f$ with period 2; from the second loop and by Lemma 2.1, there is a periodic point $z$ of $f$ with period $n$ even $n \geq 6$. Therefore, if $b c=-2$, then $\operatorname{MPer}(f)=\{1\} \cup(2 \mathbb{N} \backslash\{4\})$.

We suppose that $\mathbf{b c}=\mathbf{2}$. No odd number other than 1 belongs to $\operatorname{MPer}(f)$, as it can be seen from the examples of Fig. 32. Also from Fig. 32 we can deduce that $2 \notin \operatorname{MPer}(f)$. Now we will prove that $n \in \operatorname{Per}(f)$ for any $n$ even larger than 2 .

We know that $f$ has three basic intervals $I_{1}, I_{2}$, and $I_{3}$, the first two in $C_{1}$ and $I_{3}=C_{2}$, such that $f\left(I_{1}\right)=f\left(I_{2}\right)=C_{2}$ and $f\left(I_{3}\right)=C_{1}$ (see for instance Fig. 33). Denote $b_{0}=\inf \left\{\left(f \mid I_{3}\right)^{-1}\left(a_{0}\right)\right\}$, $a_{1}=\inf \left\{\left(f \mid I_{1}\right)^{-1}\left(b_{0}\right)\right\}$, and $b_{1}=\inf \left\{\left(f \mid I_{3}\right)^{-1}\left(a_{1}\right)\right\}$. Write $I_{1_{1}}=\left[p, a_{1}\right], I_{1_{2}}=\left[a_{1}, a_{0}\right], I_{3_{2}}$ the in- 

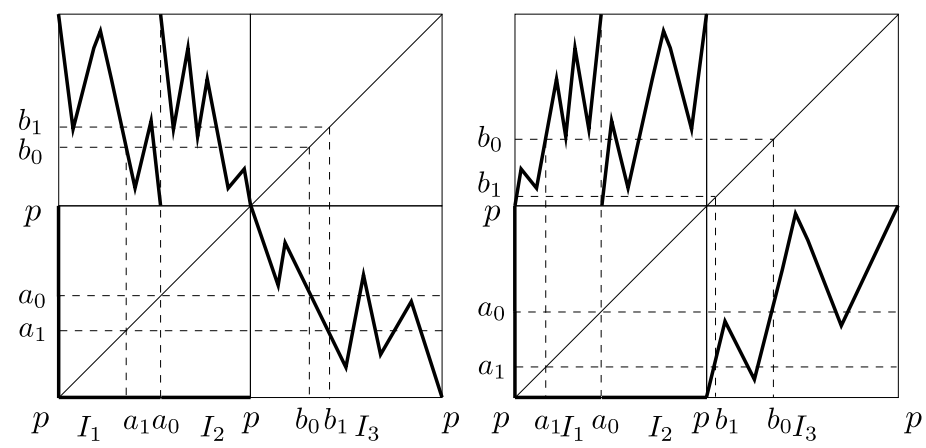

Figure 33 Examples of maps with $a=d=0$ and $b c=2$

Figure 34 Examples of maps with $a=d=0$ and either $|b|>2$ or $|c|>2$
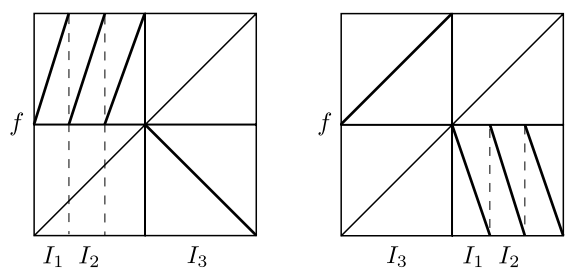

terval with endpoints $b_{1}$ and $b_{0}$, and $I_{33}$ the interval with endpoints $b_{0}$ and $p$. Then $f$ has the subgraph $I_{3_{2}} \rightarrow I_{1_{2}} \rightarrow I_{3_{3}} \rightleftarrows I_{2} \rightarrow I_{3_{2}}$. We take the non-repetitive loop $I_{2} \rightarrow I_{3_{2}} \rightarrow$ $I_{1_{2}} \rightarrow I_{3_{3}} \rightarrow I_{2} \rightarrow \cdots \rightarrow I_{3_{3}} \rightarrow I_{2}$ of length $n$ even, $n \geq 4$. By Lemma 2.1 , there is a periodic point $z$ of $f$ with period $n$ even $n \geq 4$. Therefore, if $b c=2$, then $\operatorname{MPer}(f)=\{1\} \cup(2 \mathbb{N} \backslash\{2\})$.

We consider now the case $|\mathbf{b c}|>\mathbf{2}$. We must separate the case $|b|=|c|=2$ from the others. If $|\mathbf{b}|>\mathbf{2}$ or $|\mathbf{c}|>\mathbf{2}$, then there are three basic intervals $I_{1}, I_{2}$, and $I_{3}$ such that $I_{2} \cap$ $I_{3}=\emptyset$ and $I_{1} \rightleftarrows I_{3} \rightleftarrows I_{2}$ (see for instance Fig. 34). By Lemma 2.1, the non-repetitive loop $I_{2} \rightarrow I_{3} \rightarrow I_{2}$ gives a periodic point $z$ of $f$ with period 2 , and the non-repetitive loop $I_{1} \rightarrow$ $I_{3} \rightarrow I_{2} \rightarrow I_{3} \rightarrow \cdots \rightarrow I_{2} \rightarrow I_{3} \rightarrow I_{1}$ of length $n$ even larger than 2 gives a periodic point $z$ of $f$ with period $n$ even. No odd number other than 1 belongs to $\operatorname{MPer}(f)$. Therefore, if $|b|>2$ or $|c|>2$, then $\operatorname{MPer}(f)=\{1\} \cup 2 \mathbb{N}$.

We suppose that $|\mathbf{b}|=|\mathbf{c}|=\mathbf{2}$. Clearly, no odd number other than 1 belongs to $\operatorname{MPer}(f)$. Now we will prove that $n \in \operatorname{Per}(f)$ for any $n$ even.

We know that $f$ has four basic intervals $I_{1}, I_{2}, I_{3}$, and $I_{4}$, the first two in $C_{1}$ and the others in $C_{2}$, such that $f\left(I_{1}\right)=f\left(I_{2}\right)=C_{2}$ and $f\left(I_{3}\right)=f\left(I_{4}\right)=C_{1}$ (see for instance Fig. 35). Consider $b_{1}=\inf \left\{\left(f \mid I_{3}\right)^{-1}\left(a_{0}\right)\right\}$ and $a_{1}=\inf \left\{\left(f \mid I_{1}\right)^{-1}\left(b_{1}\right)\right\}$. Denote $I_{1_{1}}=\left[p, a_{1}\right], I_{1_{2}}=\left[a_{1}, a_{0}\right]$, $I_{2}=\left[a_{0}, p\right], I_{3_{1}}=\left[p, b_{1}\right], I_{3_{2}}=\left[b_{1}, b_{0}\right]$, and $I_{4}=\left[b_{0}, p\right]$. If $(b, c) \in\{(2,2),(-2,2)\}$, then $f$ has the subgraph $I_{2} \rightleftarrows I_{4} \rightleftarrows I_{1_{2}}$. We take the non-repetitive loops $I_{4} \rightarrow I_{1_{2}} \rightarrow I_{4}$ and $I_{2} \rightarrow I_{4} \rightarrow I_{1_{2}} \rightarrow I_{4} \rightarrow \cdots \rightarrow I_{1_{2}} \rightarrow I_{4} \rightarrow I_{2}$ of lengths 2 and $n$ even larger than 2 , respectively. By Lemma 2.1, the first loop gives a periodic point $z$ of $f$ with period 2, and the second loop gives a periodic point $z$ of $f$ with period $n$ even larger than 2 . Hence, if $(b, c) \in\{(2,2),(-2,2)\}, \operatorname{Per}(f)=\{1\} \cup 2 \mathbb{N}$.

If $(b, c)=(-2,-2)$, then $f$ has the subgraph $I_{4} \rightleftarrows I_{1_{1}} \rightleftarrows I_{3_{2}}$. We consider the non-repetitive loops $I_{3_{2}} \rightarrow I_{1_{1}} \rightarrow I_{3_{2}}$ and $I_{4} \rightarrow I_{1_{1}} \rightarrow I_{3_{2}} \rightarrow I_{1_{1}} \rightarrow \cdots \rightarrow I_{3_{2}} \rightarrow I_{1_{1}} \rightarrow I_{4}$ of lengths 2 and $n$ even larger than 2, respectively. By Lemma 2.1, the first loop gives a periodic point $z$ of $f$ 


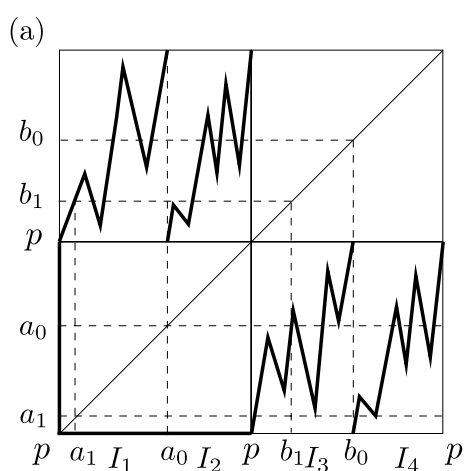

(c)

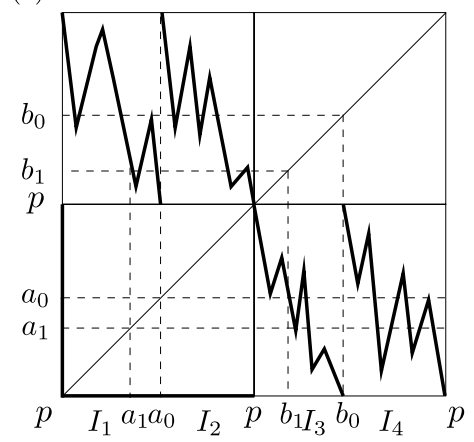

(b)

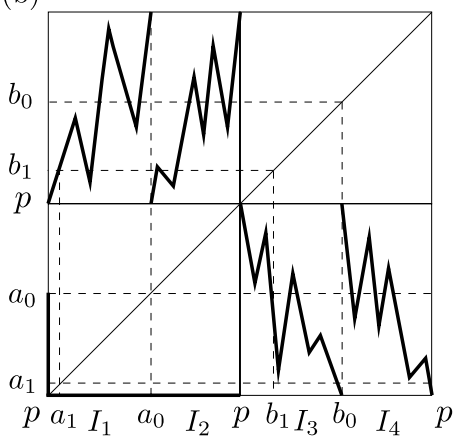

(d)

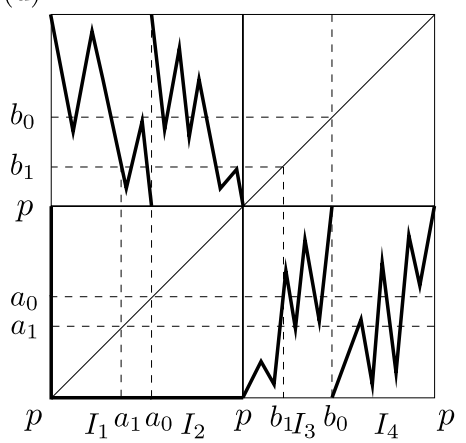

Figure 35 Examples of maps with $a=d=0$ and $|b|=|c|=2$

with period 2, and the second loop gives a periodic point $z$ of $f$ with period $n$ even larger than 2 . Hence, if $(b, c)=(-2,-2), \operatorname{Per}(f)=\{1\} \cup 2 \mathbb{N}$.

If $(b, c)=(2,-2)$, then $f$ has the subgraph $I_{4} \rightleftarrows I_{2} \rightleftarrows I_{3_{2}}$. We consider the non-repetitive loops $I_{2} \rightarrow I_{3_{2}} \rightarrow I_{2}$ and $I_{4} \rightarrow I_{2} \rightarrow I_{3_{2}} \rightarrow I_{2} \rightarrow \cdots \rightarrow I_{3_{2}} \rightarrow I_{2} \rightarrow I_{4}$ of lengths 2 and $n$ even larger than 2, respectively. By Lemma 2.1, the first loop gives a periodic point $z$ of $f$ with period 2, and the second loop gives a periodic point $z$ of $f$ with period $n$ even larger than 2. Hence, if $(b, c)=(-2,-2), \operatorname{Per}(f)=\{1\} \cup 2 \mathbb{N}$. Therefore, if $|b|=|c|=2$, then $\operatorname{MPer}(f)=\{1\} \cup 2 \mathbb{N}$. This completes the proof of statement (c3).

\section{Acknowledgements}

We thank our universities for the facilities that they gave us during the elaboration of this work

\section{Funding}

The first author has been partially supported by the Ministerio de Ciencia, Innovación y Universidades, Agencia Estatal de Investigación grants MTM2016-77278-P (FEDER), the Agència de Gestió d'Ajuts Universitaris i de Recerca grant 2017SGR1617, and the H2020 European Research Council grant MSCA-RISE-2017-777911.

\section{Availability of data and materials}

No materials are necessary.

Competing interests

The authors declare that they have no competing interests.

Authors' contributions

Both authors have contributed approximately in one half to the paper. Both authors read and approved the final manuscript.

\section{Author details}

${ }^{1}$ Departament de Matemàtiques, Universitat Autònoma de Barcelona, 08193 Bellaterra, Barcelona, Spain. ${ }^{2}$ Departamento de Matemática, Faculdade de Ciências e Tecnologia, Universidade Nova de Lisboa, 2829-516 Caparica, Portugal. 


\section{Publisher's Note}

Springer Nature remains neutral with regard to jurisdictional claims in published maps and institutional affiliations.

Received: 11 December 2019 Accepted: 2 December 2020 Published online: 18 January 2021

\section{References}

1. Abdulla, U.G., Abdulla, R.U., Abdulla, M.U., Iqbal, N.H.: Second minimal orbits, Sharkovski ordering and universality in chaos. Int. J. Bifurc. Chaos Appl. Sci. Eng. 27, 1-24 (2017)

2. Alsedà, L., Baldwin, S., Llibre, J., Swanson, R., Szlenk, W.: Minimal sets of periods for torus maps via Nielsen numbers. Pac. J. Math. 169, 1-32 (1995)

3. Alsedà, L., Juher, D., Mumbrú, P.: On the preservation of combinatorial types for maps on trees. Ann. Inst. Fourier (Grenoble) 55(7), 2375-2398 (2005)

4. Alsedà, L., Llibre, J., Misiurewicz, M.: Combinatorial Dynamics and Entropy in Dimension One, 2nd edn. Advanced Series in Nonlinear Dynamics, vol. 5. World Scientific, Singapore (2000)

5. Alsedà, L., Ruette, S.: Rotation sets for graph maps of degree 1. Ann. Inst. Fourier (Grenoble) 58(4), 1233-1294 (2008)

6. Arai, T.: The structure of dendrites constructed by pointwise P-expansive maps on the unit interval. Discrete Contin. Dyn. Syst. 36, 43-61 (2016)

7. Bernhardt, C.: Vertex maps for trees: algebra and periods of periodic orbits. Discrete Contin. Dyn. Syst. 14(3), 399-408 (2006)

8. Bernhardt, C.: Vertex maps on graphs-trace theorems. Fixed Point Theory Appl. 2011, 8 (2011)

9. Block, L., Guckenheimer, J., Misiurewicz, M., Young, L.S.: Periodic points and topological entropy of one dimensional maps. In: Global Theory of Dynamical Systems. Lecture Notes in Math., vol. 819, pp. 18-34. Springer, Berlin (1980)

10. Brown, R.F:: The Lefschetz Fixed Point Theorem. Scott, Foresman, Chicago (1971)

11. Casasayas, J., Llibre, J., Nunes, A.: Periodic orbits of transversal maps. Math. Proc. Camb. Philos. Soc. 118, 161-181 (1995)

12. Efremova, L.S.: Periodic orbits and the degree of a continuous map of a circle. Differ. Integer Equ. (Gor'kiī) 2, 109-115 (1978) (in Russian)

13. Jiang, B.: Lectures on Nielsen Fixed Point Theory. Contemporary Mathematics, vol. 14. Am. Math. Soc., Providence (1983)

14. Jiang, B., Llibre, J.: Minimal sets of periods for torus maps. Discrete Contin. Dyn. Syst. 4, 301-320 (1998)

15. Kiang, T.H.: The Theory of Fixed Point Classes. Springer, Berlin (1989)

16. Llibre, J.: Periodic points of one dimensional maps. In: Mira, C., Netzer, N., Simó, C., Targonski, G. (eds.) European Conference on Iteration Theory, ECIT 89, pp. 194-198. World Scientific, Singapore (1991)

17. Llibre, J., Misiurewicz, M.: Negative periodic orbits for graph maps. Nonlinearity 19(3), 741-746 (2006)

18. Llibre, J., Sá, A.: Periods for continuous self-maps of a bouquet of circles. C. R. Acad. Sci. Paris Sér. I Math. 318, 1035-1040 (1994)

19. Los, J.E., Nitecki, Z.H.: Edge-transitive graph automorphisms and periodic surface homeomorphisms. Discrete dynamical systems. Int. J. Bifurc. Chaos Appl. Sci. Eng. 9(9), 1803-1813 (1999)

\section{Submit your manuscript to a SpringerOpen ${ }^{\circ}$ journal and benefit from:}

- Convenient online submission

- Rigorous peer review

- Open access: articles freely available online

- High visibility within the field

- Retaining the copyright to your article

Submit your next manuscript at $\gg$ springeropen.com 